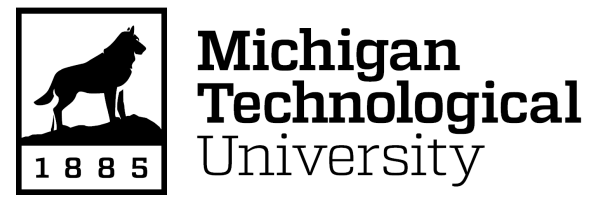

Michigan Technological University Digital Commons @ Michigan Tech

Dissertations, Master's Theses and Master's Reports

2018

\title{
A Bluetooth Low-Energy Wireless Sensor Platform for Continuous Monitoring of a Bioreactor Environment during Cell Manufacturing
}

Brad Nelson

Michigan Technological University, bnelson@mtu.edu

Copyright 2018 Brad Nelson

Recommended Citation

Nelson, Brad, "A Bluetooth Low-Energy Wireless Sensor Platform for Continuous Monitoring of a Bioreactor Environment during Cell Manufacturing", Open Access Master's Thesis, Michigan Technological University, 2018.

https://doi.org/10.37099/mtu.dc.etdr/609

Follow this and additional works at: https://digitalcommons.mtu.edu/etdr

Part of the Biomedical Devices and Instrumentation Commons 
A BLUETOOTH LOW-ENERGY WIRELESS SENSOR PLATFORM FOR CONTINUOUS MONITORING OF A BIOREACTOR ENVIRONMENT DURING CELL MANUFACTURING

\author{
By \\ Bradley D. Nelson
}

\begin{abstract}
A THESIS
Submitted in partial fulfillment of the requirements for the degree of MASTER OF SCIENCE

In Electrical Engineering
\end{abstract}

MICHIGAN TECHNOLOGICAL UNIVERSITY

2018

(C) 2018 Bradley D. Nelson 
This thesis has been approved in partial fulfillment of the requirements for the Degree of MASTER OF SCIENCE in Electrical Engineering.

Department of Electrical and Computer Engineering

$\begin{aligned} \text { Thesis Advisor: } & \text { Keat Ghee Ong } \\ \text { Committee Member: } & \text { Paul Bergstrom } \\ \text { Committee Member: } & \text { Feng Zhao } \\ \text { Department Chair: } & \text { Daniel R. Fuhrmann }\end{aligned}$




\section{Table of Contents}

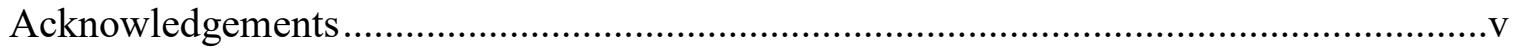

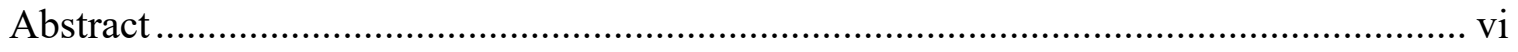

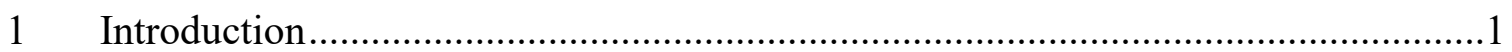

Wireless Sensor Technology .................................................

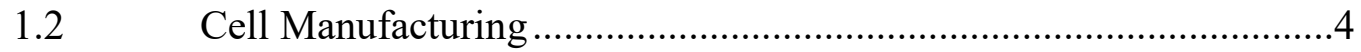

The Bioreactor Environment ........................................................

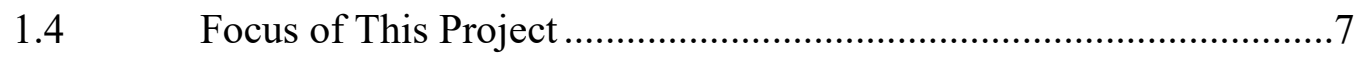

2 Sensor Platform Design and Fabrication .....................................................

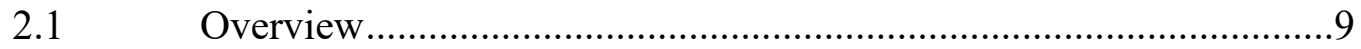

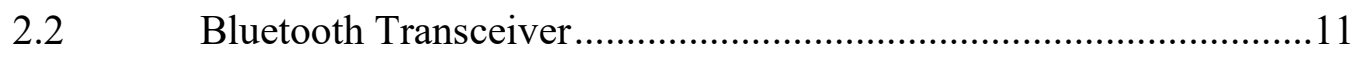

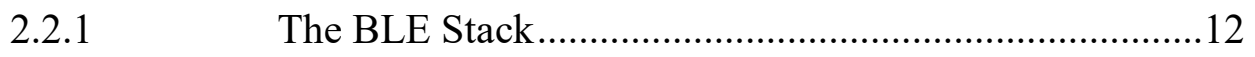

2.2.2 GATT Profile..............................................................24

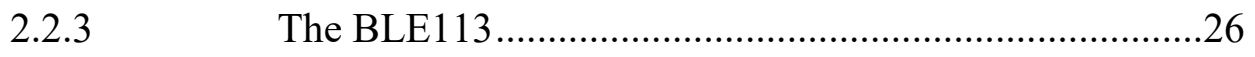

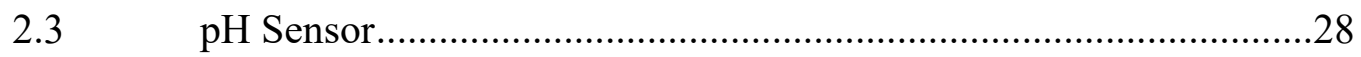

2.3.1 Electrode Design.................................................28

Signal Processing .......................................................

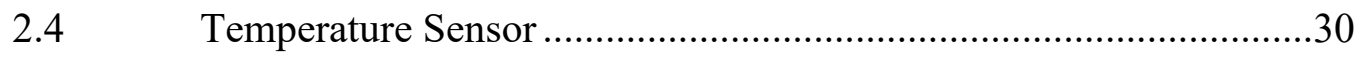

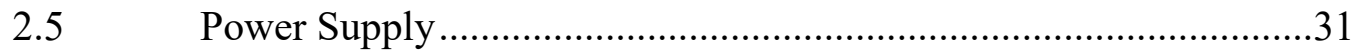

2.5.1 Battery Selection......................................................32

2.5.2 Wireless Battery Charging ........................................33

2.5.3 Wireless Power Receiver Design................................35

2.5.4 Wireless Power Transmitter Compatibility ...................37

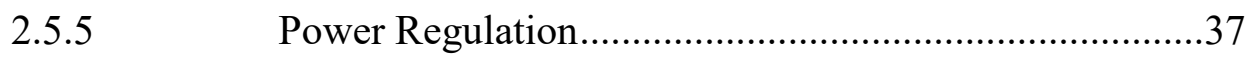

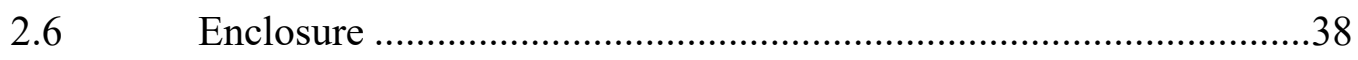


2.7 Operation of the Wireless Sensor

2.8 Access Point Software

3 Validation Experiments

$3.1 \quad$ Methods

\section{1 .1}

pH Characterization

3.1 .2

Response Time Characterization

3.2

Results 50 


\section{Acknowledgements}

I would like to thank Prof. Keat Ghee Ong for his role as a mentor. He has always been there to push me to achieve more than I would push myself, while also always being available as a guide and resource. I greatly respect him for his flexibility and for his concern that I work on projects that I actually find interesting.

I would also like to thank Salil Sidharthan Karipott and the other students in my lab for their support in all the work I have done, and for always being around to bounce ideas off of.

Finally, I would like to thank my family for their constant support and encouragement, even when I am far from home. 


\section{Abstract}

A wireless sensor platform based on Bluetooth Low-Energy (BLE) technology was designed and prototyped for continuous monitoring of physical conditions and chemical analytes, which could be applied to bioreactors during the cell manufacturing process. Controlling environmental conditions such as $\mathrm{pH}$, oxygen, glucose, temperature, and pressure is vital to ensure the consistency of the manufactured cells and maintain the potency of the product. Current methods to control bioreactor conditions focus only on monitoring the cell culture environment during cell growth, but there is a lack of direct quantification of cell properties to provide an integrated feedback system that can also maintain cell quality. Furthermore, current methods are typically expensive and inflexible for new bioreactor designs. The ultimate goal of this project is to develop a low-cost wireless sensor platform that can incorporate different types of sensors for monitoring both growth conditions and cell quality in various types of bioreactors.

This thesis represents the first phase of the project with the development of the sensor platform and prototyping a $\mathrm{pH}$ and temperature sensor module along with the platform. Bench tests demonstrated the efficacy of these sensors in continuous monitoring of $\mathrm{pH}$ and temperature over several days. With the sensor functionality proven, the next step is to examine the biocompatibility of the sensor, as well as expand the parameters to include oxygen, glucose, and pressure. New sensors, such as those based on the impedimetric technique, will also be developed to direct cell quality evaluation. 


\section{Introduction}

\subsection{Wireless Sensor Technology}

Sensors are ubiquitous in the world around us. They are used for diverse applications in many fields including agriculture [1,2], health and fitness [3], transportation [4], military [5], structural monitoring [6], and space research [7, 8]. In addition to gathering information that can improve our daily life (e.g., sensors used for weather forecast), these sensors provide critical data needed for many devices and equipment to function properly (e.g., oxygen sensors or collision detection sensors used in our cars). Without them, much of the technology that drives the world we live in could not exist.

A wireless sensor is typically an electronic device that can gather local information and then transmit the measurements to a remote reader/receiver via wireless means (radio waves, magnetic fields, etc.). Without the need of wire connections, these sensors are convenient to deploy, are highly adaptive and can be replaced and reconfigured easily. Today, instead of deploying a single sensor, many sensing applications rely on an array of sensors capable of monitoring different parameters over a large area. These sensors are interconnected wirelessly via networked communications, and together they are referred to as a wireless sensor network.

Within a wireless sensor network, a single wireless sensor is commonly referred to as a node. A node usually contains one or more sensors, a transceiver, controller, memory storage, and power supply [9, 10]. Many wireless sensor network designs are built upon an existing platform. For example, the Mica platform [11] was originally developed at Berkeley in the early 2000's. The platform was designed with flexibility in mind, and boasted a battery life that exceeded other portable technologies like cellphones. The Mica mote contains a 51-pin header which allows for the user to connect their choice of sensing peripherals to the platform. A competing platform, the Telos, [12] is very similar in design to the Mica motes, but prioritized for minimal current consumption. Furthermore, only 16 pins are available on the Telos for external sensors, thus it cannot support as many sensors as the Mica platform. A more recent system, Shimmer ${ }^{\mathrm{TM}}$ [13], was designed with the biomedical field specifically in mind. As a result, the platform was smaller. All available 
sensors for the Shimmer ${ }^{\mathrm{TM}}$ platform are integrated into the packaging to minimize the footprint of the device.

Three useful categories of wireless sensor networks are: broadcast networks, point-topoint networks, and mesh networks (Figure 1.1). A broadcast network features unidirectional communication from a single node, usually called a beacon. This beacon node repeatedly transmits its data to any number of devices in range without forming a link. A point-to-point network contains bidirectional communication between nodes. A point-to-point network may feature more than two or more nodes, where the communication of each node is only destined for direct neighbor nodes. The two nodes establish a wireless link, and may communicate back and forth, but only communicate with each other until the link is severed. In a mesh network, each node may form links with several adjacent nodes, and information may be relayed through nodes that are not the destination node. Different network topologies are better suited for different applications, and none is necessarily better than another.

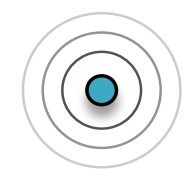

Broadcast

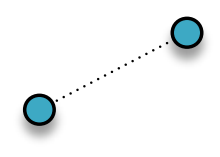

Point-to-point

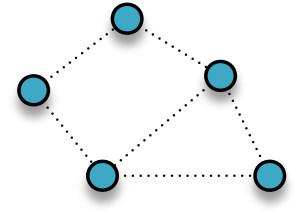

Mesh

Figure 1.1. Wireless sensor network topologies.

The method of communication between wireless sensor nodes is a significant consideration. Most wireless sensor networks are considered to be wireless personal area networks (WPAN) and the communication protocols are governed by the IEEE 802.15 standards [14]. These standards describe the use of the medium, which provides basic constraints for the lowest layers of the wireless sensor node: the PHY (physical) layer and the MAC (medium access control) layer. The PHY layer refers to how the radio wave is shaped. This includes the frequency, bandwidth, modulation, and similar concerns. The MAC layer refers to when a device is allowed to communicate, including what to do when multiple devices try to communicate at the same time. 
Two general communication styles exist within the IEEE 802.15 standards. Nodes may interact using high data rate communication in which a wide bandwidth is used to transfer large amounts of data in short periods of time. Alternatively, the standards describe a low data rate communication in which a narrow bandwidth is used to transmit smaller packets of data. Low data rates are often preferred in wireless sensor networks due to the limited power supply of most wireless sensor nodes. The Bluetooth Low-Energy (BLE) and ZigBee protocols are common low data rate options, while the Bluetooth Enhanced Data Rate (EDR) commonly used in music streaming applications lean in the direction of high data rate communication.

Larger and more complex network topologies introduce new considerations into wireless sensor networking. The broadcast topology may be considered a network of a single node because only the beacon is using the medium. There may be any number of receiver devices, but they do not add to the complexity of the network. Point-to-point topologies introduce a second communicating device and require the addition of a link layer. This link layer manages which devices are connected and which are not. There is often a specified relationship between linked nodes, such that a slave node receives orders from the master or a server node stores the data from the client. Mesh topologies require this link layer but also introduce a routing layer to specify how devices in the same network that are not linked can communicate with each other.

Wireless sensors have seen significant use in the medical field. Cumbersome needleor probe-based transducers [15] have been largely replaced by wireless sensor technology $[16,17]$. Implantable wireless sensors have been used in the field of orthopedics to monitor the forces acting on bones and joints [17-19]. Wearable sensors have also been used to provide a non-invasive alternative in many patient monitoring applications. These wearable wireless sensors typically measure various parameters through tissues [20] or fluids $[21,22]$.

The sensor platform described in this work has been designed for use in a wide assortment of applications. The platform is small enough to fit the needs of some implantable or wearable sensor needs, as well as for monitoring the operating conditions and product potency of the cell manufacturing process. The use of larger motes like the 
Mica or Telos is not appropriate. Therefore, a new design was needed. In order to minimize the size of the platform, sensors built using this platform are built-in for particular applications without additional modules.

\subsection{Cell Manufacturing}

Cell manufacturing is the process of growing biological tissue ex vivo. This includes engineering of tissues and organs as well as large quantities of a single type of cell. The eventual goal of this work focuses on immunological cell manufacturing, with the application of chimeric antigen receptor (CAR) modified T-cells in mind. The manufacture of CAR T-cells specifically refers to the creation of T-cells with engineered CAR proteins which will target and destroy certain cancerous tumors.

T-cell therapy has been shown to be effective in the remission of melanoma [23, 24] and leukemia $[25,26]$. These therapies utilize ex vivo cell manufacturing processes, which require careful monitoring to ensure that the product has the desired potency.

Current methods of cell manufacturing involve a lengthy and expensive multi-step process [27-30]. This process (Figure 1.2) may generally be described as containing (1) apheresis, (2) selection, (3) activation, (4) transduction, (5) expansion, (6) storage, and (7) infusion. Apheresis is the removal of blood from the body of a patient or donor in order to extract the desired leukocytes [31]. Different cells are separated and the selected T-cells are removed and washed. In the activation step, dendritic cells or B-cells may be used to present the T-cells with the target antigens, but this method is rarely cost or time effective. Instead, artificial antigen-presenting cells (aAPC) in the form of small beads are usually introduced to the T-cell suspension. The cell transduction step usually occurs at the same time as activation, but it involves the introduction of a genetic viral vector to the suspension which leads to the production of synthetic CAR proteins. Cells are then moved to a bioreactor for expansion, which takes 9-11 days. There are two distinct methods used in expansion. In batch suspension culture, no new materials are introduced to the culture

during the manufacturing process, while in batch-fed suspension culture new substances are periodically introduced [32]. After the expansion process is complete, the aAPCs are 
removed and the product cells are cryogenically stored until they are finally infused during the treatment of the patient.

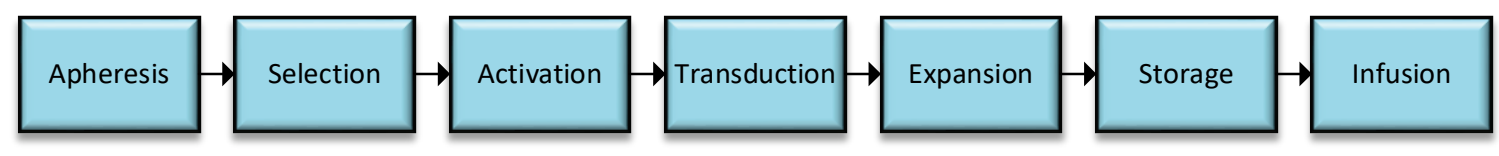

Figure 1.2. The cell manufacturing process.

This process has several limitations, including expense, scalability, and potency. T-cell therapy is very expensive due to the need for proper facilities, equipment, and trained personnel [33]. Novartis, the first company to receive FDA approval for the treatment, has announced a price of $\$ 475,000$ for a single infusion and estimate that the total treatment will be around $\$ 574,000$ [34]. The manufacture of CAR T-cells is currently limited to small-scale manufacture, and significant action must be taken to allow the large-scale manufacture of the treatment [35]. It is difficult to create uniformly potent treatments at different facilities because every step in the manufacturing process in which the cells are sampled introduces the risk of contamination [28]. But without sampling, the potency of the cells cannot be judged and the conditions of the manufacturing process cannot be modified to improve growth [36].

The system described in this work seeks to address these limitations by providing a low-cost, in-line sensor platform that can observe the conditions of the cells during the long expansion stage and report those conditions in real time. This will reduce expense by providing an alternative to the large, expensive bioreactors tailored to the needs of T-cell manufacturing for smaller institutions. The system will provide the ability to monitor the status of a large network of bioreactors so that the expansion process can be performed for multiple patients simultaneously with minimal personnel overhead. The in-line nature of the system eliminates the need for samples to be taken throughout the expansion process, reducing the risk of contamination of the culture.

\subsection{The Bioreactor Environment}

During the expansion phase, growing cells are nurtured in a carefully controlled environment within a bioreactor. For cells to thrive, a sufficient quantity of oxygen must be present in that environment. Therefore, healthy concentrations and solubilities of both 
$\mathrm{O}_{2}$ and $\mathrm{CO}_{2}$ must be maintained. Some of the most important parameters to control in the bioreactor include $\mathrm{pH}, \mathrm{dO}_{2}$, temperature, and pressure [37]. During the growth process, cells consume glucose and release lactate into the environment. If this environment is not carefully maintained it may become hazardous to the cells [38].

Various methods exist to monitor these vital parameters. It is common for many of these values to be sampled intermittently during the expansion process by the operator. However, this is undesirable because it exposes the cell culture to foreign contaminants [28]. Therefore, there is a need for methods to monitor the suspension conditions without exposing the bioreactor to unnecessary contaminants. Several bioreactors are marketed which monitor the cellular environment in-line. Examples of this include the G-Rex ${ }^{\circledR}$ (Wilson Wolf) and the CliniMACS Prodigy ${ }^{\circledR}$ (Miltenyi Biotec). Such systems are highly desirable, but their high cost and limited flexibility may make them inaccessible to a wide range of institutions. This often results in a lack of standardization between institutions, which has been observed to accelerate the development of new treatments, particularly during the early stages [37]. If there is to be comparison between procedures performed by different institutions, those institutions must have the tools to adequately quantify the conditions of the cell culture during their procedures. Therefore, such tools must be available not only for large-scale industrial bioreactors, but also for benchtop bioreactors in the lab.

One promising technology for bioreactor monitoring is the use of spectroscopy. The appeal of the spectroscopic method is that measurements may be made optically through the walls of the bioreactor, eliminating the contamination caused by manual sampling. Several forms of spectroscopy - including near-infrared [39], mid-infrared [40], Raman [41], and fluorescence [42] — have been demonstrated in bioreactors or similar conditions. However, the use of spectroscopy is plagued with low selectivity [43]. Fluorescence spectroscopy may improve selectivity to some extent by using fluorescent tags in the cells of interest, but the use of foreign tags may not be desirable for certain cell manufacturing protocols. Therefore, there is a need for a tag-less, low-cost sensor system with greater selectivity. 
An alternative to the spectrometric method is to implement a device which can monitor the conditions inside the bioreactor with using a wireless sensor and transmit the results to a remote device. This may be accomplished using passive sensors such as a $\mathrm{pH}$ sensitive resonant sensor [44] which may be interrogated remotely. Active systems may also be used, which can provide greater flexibility in measuring multiple parameters simultaneously [45]. In situ sensors are able to provide the robust representation of the cellular environment found in on-line monitoring in expensive bioreactors with reduced cost and added flexibility. Wireless sensors in particular may provide this without the need to physically access the bioreactor during its operation, significantly reducing the risk of contamination. However, there has not yet been a wireless sensor of sufficiently small size to monitor multiple parameters inside the bioreactor environment.

\subsection{Focus of This Project}

This work is the first phase of the development of a wireless sensor node for cell manufacturing. The aim of this first phase is to demonstrate the viability of the sensor platform. A sensor platform will be developed for real-time monitoring and transmission of temperature and $\mathrm{pH}$ data in a liquid environment. To achieve this, a miniature $\mathrm{pH}$ sensor, temperature sensor, and fabricated enclosure will be created and tested. However, the creation of a miniature $\mathrm{pH}$ sensor or packaging are not the goal of this work.

The sensor node developed by this work must meet several requirements (Table 1.1). The sensor must communicate wirelessly to an external device so that the device may perform online monitoring without the need to expose the contents of the bioreactor to contamination. The minimum range of this communication needs to be sufficient to reach a nearby computer, at least $5 \mathrm{~m}$. The sensor must be small enough to be contained in a benchtop bioreactor without impeding the action of the mixer. Using the Wheaton ${ }^{\circledR} 500$ $\mathrm{mL}$ Celstir Complete as a representative $500 \mathrm{~mL}$ benchtop bioreactor, an appropriate size for the sensor that will not impede mixing will be approximately $30 \mathrm{~mm}$ x $30 \mathrm{~mm}$ x 20 $\mathrm{mm}$. The power supply for the sensor must supply $2.5 \mathrm{~V}$ for 2 weeks to exceed the 9-11 day expansion phase. This sensor will be disposable so that differing sterilization protocols at different institutions will not be a source of discrepancy. Therefore, the cost of the sensor 
should be kept to a minimum. At this stage, the expected sensor material cost is set at US\$50. Based on the typical price of cell lines used in cell manufacturing, which typically range from a few hundreds to thousands, a disposable sensor of less than $\$ 100$ should still be economically viable for cell manufacturing if it can ensure the quality of cells.

Table 1.1. Specifications for the sensor platform to monitor bioreactor conditions.

\begin{tabular}{|r|c|}
\hline Requirements & Specifications \\
\hline Wireless communications & $5 \mathrm{~m}$ \\
\hline Size & $30 \mathrm{~mm} \times 30 \mathrm{~mm} \times 20 \mathrm{~mm}$ \\
\hline Power requirement & $2.5 \mathrm{~V}$ \\
\hline Continuous operation & 2 weeks \\
\hline Unit cost & $\$ 50 \mathrm{USD}$ \\
\hline One time use & Yes \\
\hline
\end{tabular}




\section{Sensor Platform Design and Fabrication}

\subsection{Overview}

To address the need for wireless communication indicated in Table 1.1, a wireless sensor node is used for communication and operation control. The sensor continuously monitors the acidity and temperature of cells within the expansion medium, and other parameters will be included in the subsequent projects. This data is transmitted wirelessly in real time using Bluetooth Low-Energy (BLE) communication to a hub connected to a computer which will store and display the results. The advantage of using BLE is that several small, low-cost BLE microcontrollers are already commercially available, and their cost range is low enough for this sensor to be used on a disposable basis (the BLE used in this project is about USD $\$ 11$ when purchased at low quantity). BLE also offers robust connections so that communication between devices may continue over the course of a two-week expansion process. The maximum transmission range of BLE is typically around $100 \mathrm{~m}$ in air, though this may vary based on the power supply and antenna configuration. If longer transmission range is needed, a BLE mesh network will be implemented to extend the range of these sensor nodes.

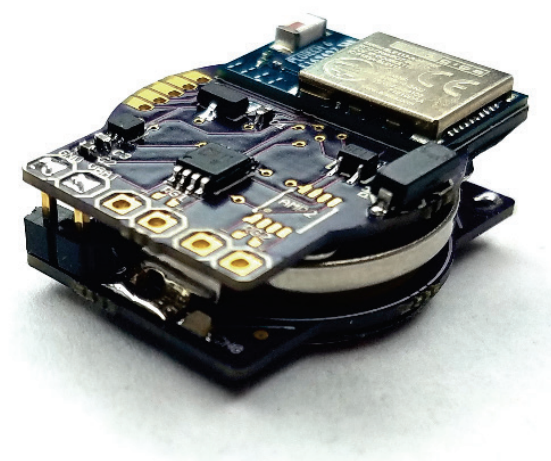

Figure 2.1. Sensor platform.

One of the most significant concerns in the development of wireless sensors is power consumption. Wireless sensors typically have a limited supply of power which must be conserved to maximize the lifetime of the sensor. Data transmission is the greatest drain on the battery of a wireless sensor, so the amount of time that the sensor spends transmitting 
must be kept at a minimum. Some peripheral sensing hardware, such as signal amplifiers and resistive sensors, also contribute to battery drain. Therefore, to achieve the 2-week requirement, a wirelessly rechargeable battery system has been incorporated into the sensor node.

The hardware of this wireless sensor node is represented in Figure 2.2. The communication device, controller, and memory are all within the Silicon Labs BLE113 module, and are discussed in Section 2.2. The sensors, along with the associated signal processing electronics, are discussed in Sections 2.3 and 2.4. The power supply is discussed in Section 2.5.

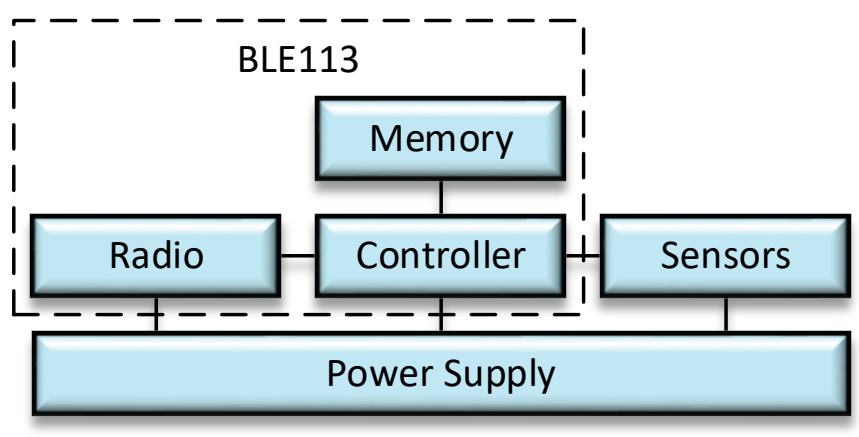

Figure 2.2. Hardware components of the wireless sensor node.

The physical design of the sensor node is shown in Figure 2.3. To limit the footprint of the device to $30 \mathrm{~mm} \times 30 \mathrm{~mm}$ (see Table 1.1), a multi-layer design featuring two printed circuit boards has been implemented. One side of the sensor node features the BLE113 as well as the sensors and signal processing circuits. The other side contains an inductive charging coil, wireless power receiver, and battery charger. A rechargeable lithium-ion battery is located between the two boards. The enclosure for the sensor node is described in Section 2.6. 


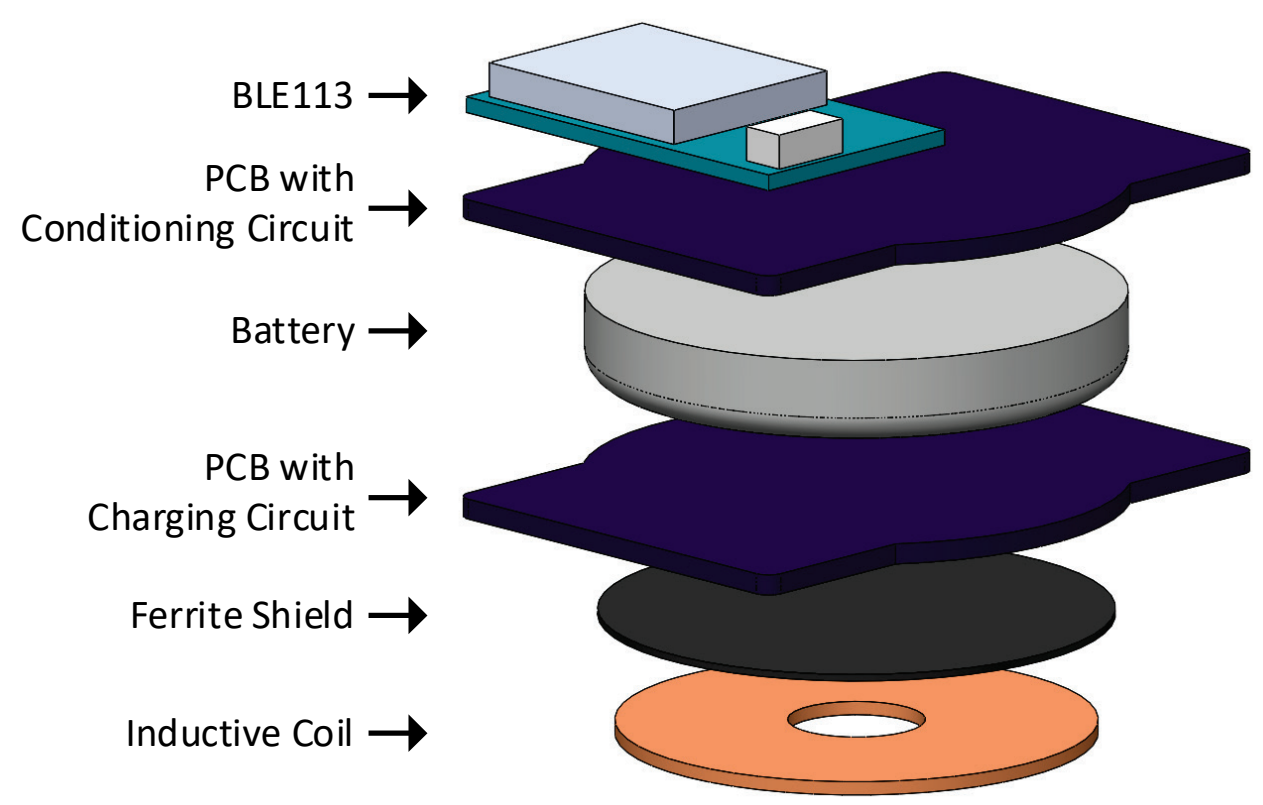

Figure 2.3. Sensor node design.

\subsection{Bluetooth Transceiver}

Bluetooth has become a household name in the field of wireless communication. When the technology was first conceived, the creators imagined a future world free from wires. They planned to create a technology that would replace wires in many areas. It was not the first form of wireless communication, as it was preceded by technologies like radio communication and Wi-Fi. What made Bluetooth distinctly different from other technologies available at its release was the operation using short-range, point-to-point connections. This makes the technology better suited to many wireless sensor networking applications than its predecessors.

Many people use Bluetooth technology daily as their phones are paired with their cars, wearable devices, and portable speakers. The needs of a device that streams audio versus a device that occasionally transmits sensor data are very different. Wireless communication requires significant amounts of power but wireless devices, whether cell phones or wireless sensors, operate using a limited power supply. In order to suit the technology for different applications, the Bluetooth standards have introduced several different physical and MAC layers. These different standards are called volumes. Bluetooth 2.0 introduced an Extended 
Data Rate (EDR) volume to improve the data rate for audio transmission. Bluetooth 3.0 introduced an Alternative MAC/Physical (AMP) volume as another high-speed platform based on the 802.11 standards. Bluetooth 4.0 ushered in the Bluetooth Low-Energy (BLE) volume which features a decreased data rate to minimize power consumption for low power devices like wireless sensors. This, in addition to revisions to Bluetooth 5.0 that include support for mesh networking, has made Bluetooth wireless sensor networking a viable option.

\subsubsection{The BLE Stack}

The Bluetooth specifications describe the stack structure using two volumes: the Controller and the Host. All Bluetooth devices share the same Host volume, but there are currently three different Controller volumes available: the Basic Rate and Extended Data Rate (BR/EDR) Controller, the Low-Energy (LE) Controller, and the Alternative MAC/Physical (AMP) Controller. A Bluetooth device includes the Host volume as well as one or more Controller volumes. A primary controller, containing either the LE Controller, $\mathrm{BR} / \mathrm{EDR}$ Controller, or both, is always present. Additionally, one or more AMP Controllers may be included. The platform described in this work utilizes the Bluetooth Low-Energy (LE) Controller volume described in the Bluetooth 4.0 specifications as the entire Controller volume.

The Bluetooth stack, shown in Figure 2.4, is comprised of the Controller and Host volumes, as well as a transport layer between them called the Host-Controller Interface (HCI). The Application Layer exists above the Host and is not part of the Bluetooth stack. The Controller volume contains the Physical Layer (PHY) and Link Layer (LL). The Host volume contains the Logical Link Control and Adaptation Protocol (L2CAP), the Attribute (ATT) Protocol and its extension the Generic Attribute Profile (GATT), the Service Discovery Protocol (SDP), the Security Manager Protocol (SDP), and the Generic Access Profile (GAP). A Transport Layer called the Host Controller Interface (HCI) exists between the Host and Controller volumes. 


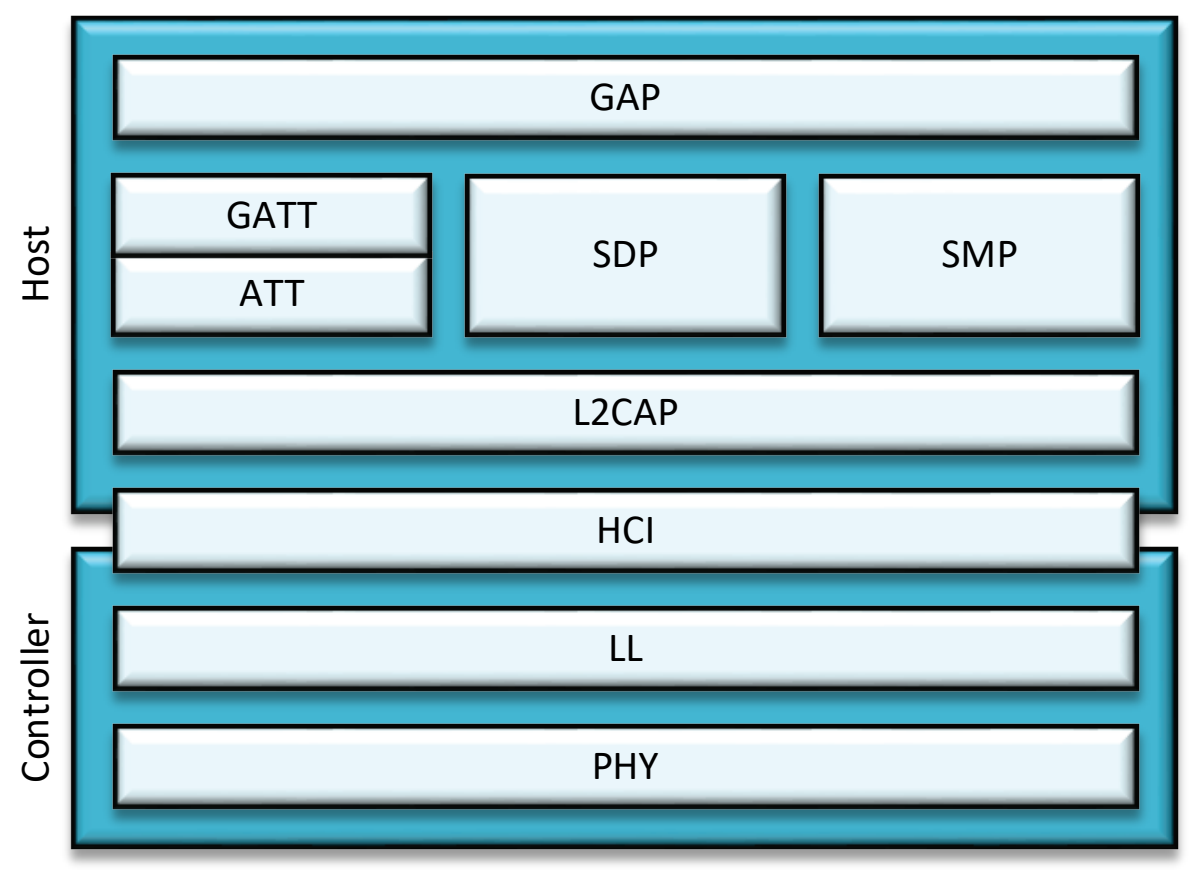

Figure 2.4. The Bluetooth Low-Energy stack.

Physical Layer (PHY): The Physical Layer of the Bluetooth specifications describes the frequencies that will be used by Bluetooth wireless communication. Bluetooth devices operate in the ISM band between the frequencies of 2.400 and $2.4835 \mathrm{GHz}$. In the LowEnergy Controller volume, this band is divided into 40 channels. Three of these channels are reserved specifically for advertising packets, while the remaining 37 are used to transmit data. Data is modulated using Gaussian Frequency Shift Keying (GFSK).

The use of the $2.4 \mathrm{GHz}$ band may be questioned for a submerged device, as path loss has been observed to be less extreme at lower frequencies in underwater environments [46]. However, the $2.4 \mathrm{GHz}$ band is frequently used in underwater sensor networks due to its increased data rate [47]. In this application, the implementation of Bluetooth technology allows greater ease of integration into existing systems.

Link Layer (LL): The Link Layer describes how point-to-point Bluetooth communicate with each other. This is done by pairing devices, a process of forming and maintaining connections between nearby devices. The Bluetooth Link Layer is composed of three components (shown in Figure 2.5): the Link Manager, the Baseband Resource Manager, and the Link Controller. 


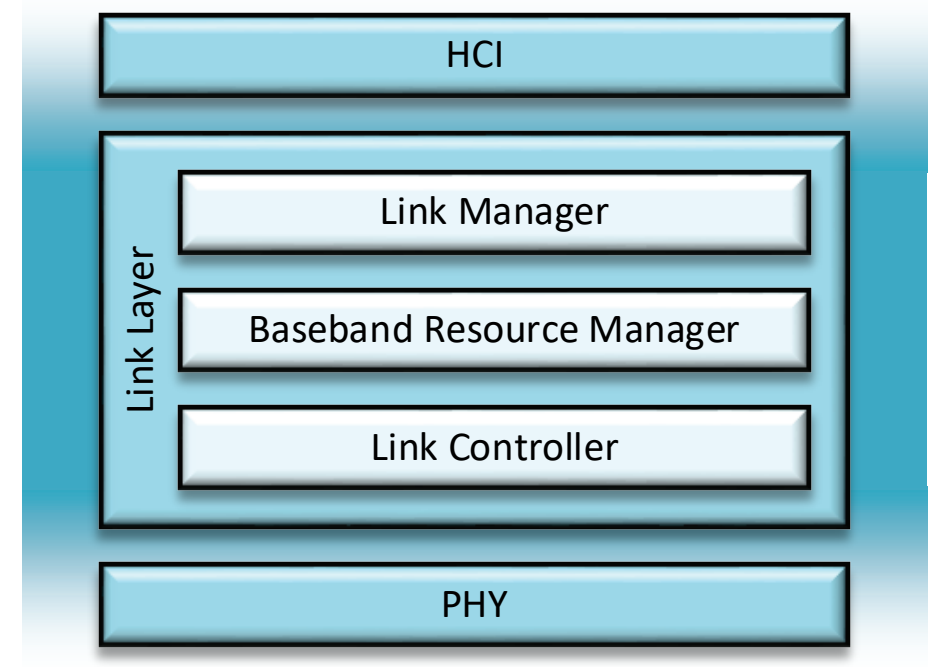

Figure 2.5. Link Layer structure.

The Link Manager is responsible for the creation, upkeep, and removal of links between devices. Bluetooth devices may be in one of five states, shown in Figure 2.6. Devices in the Standby state do not communicate. From here, a device may enter the Advertising state in which it repeatedly broadcasts data to all nearby devices. Advertisers may broadcast their name, address, and a list of services it can perform so that they may be recognized by other devices. A device may also enter the Scanning state; in which it listens to nearby advertisers. When a scanner has identified an advertiser that it wants to connect to, it may enter the Initiating state where it begins communicating with the advertiser to form a connection. When this process is successful, both initiator and advertiser enter the Connected state, and a connection is formed. 


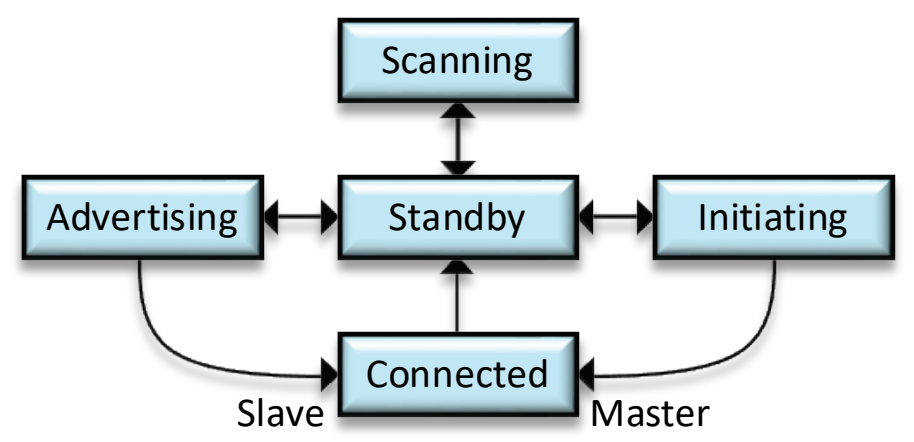

Figure 2.6. Device states.

The Bluetooth specifications use several pairs of roles to describe how devices interact. Connected devices have the master/slave roles. The initiator becomes the master, and is responsible for managing the clocks of all slaves connected with it and for managing the adaptive channel hopping during communication. The advertiser becomes the slave. Other roles used within the link layer context include the central and peripheral. The master has the central role before the connection is established, so that a device that is scanning or initiating may be the central. The slave has the peripheral role before connection, so that an advertiser may have the peripheral role. In broadcast networks, central and peripheral roles are replaced with observer and broadcaster, respectively.

The Link Layer Control Protocol manages connected devices. When the new connection is initiated, the slave is given an interval during which it must confirm that it is still connected and a latency specifying the number of intervals it may miss. If the slave fails to maintain the connection, a link loss error occurs and the connection is broken. This protocol is also used to maintain a map of the frequency channels currently in use by nearby devices.

The Baseband Resource Manager controls access to the medium, which is specified by the Air Interface Protocol. Multiple advertising and data channels are used to provide multiple access to the medium using different times and frequencies. Different clock accuracies for active and sleep cycles are enforced by the master. Unconnected devices transmit packets with randomized delays to prevent collisions with other devices. Frequency division is achieved using adaptive channel hopping within the data channels. The adaptive channel hopping algorithm provides the slave with the channel to use for transmission and reception while avoiding bad channels. Any or all of the three advertising 
channels may be used, as coordinated by the Host volume. In addition, specific devices can be whitelisted by the host to limit the number of transmissions a device will respond to.

The Link Controller governs packet transmission and signaling between devices. Bluetooth standards also specify bitstream processing for transmission and reception. Packets received from the Link Layer have the structure shown in Figure 2.7 featuring a preamble, address, payload, and CRC. The header contained in the payload differs in structure across the advertising and data channels, but the general payload structure remains the same. Data packets may contain an optional Message Integrity Check (MIC) field at the end of the payload. All packets in the bitstream undergo encryption/decryption, CRC generation/checking, and whitening/dewhitening.

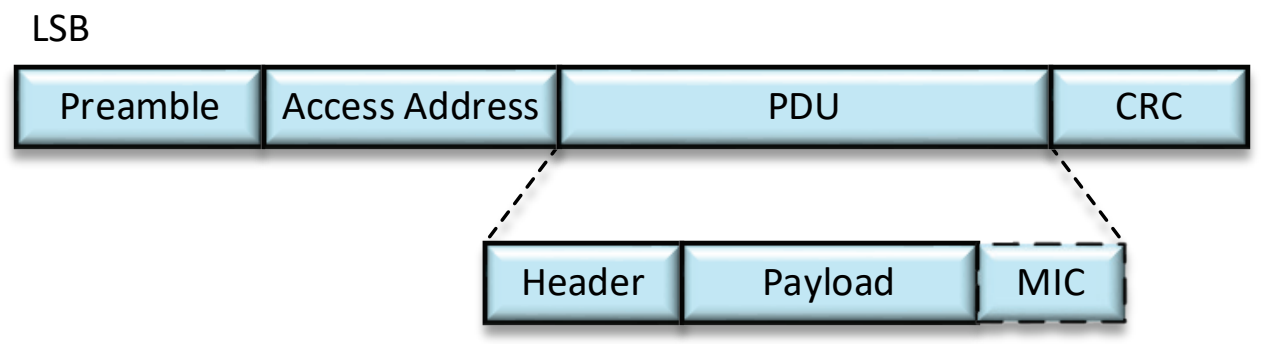

Figure 2.7. Link layer packet structure.

The formation of links provides a bidirectional connection between the peripheral device inside the bioreactor and a central device which receives transmitted data. This bidirectional connection allows the device within the bioreactor to be controlled remotely, thus removing the need to open the bioreactor to external contamination.

Host Controller Interface (HCI): The Host Controller Interface provides several methods for the Bluetooth Controller to communicate with a Host. In many cases, the Controller volume is implemented using hardware while the Host volume exists in a microcontroller or computer. This separation allows for the Host to sleep while the Controller remains active. The Host Controller Interface provides a consistent method for different types of Host volumes to interact with any of the available Controller volumes over multiple interfaces such as USB, UART, and RS232. Commands pass from the Host to the Controller, while events and data packets pass from the Controller to the Host.

Logical Link Control and Adaptation Protocol (L2CAP): The L2CAP layer is the lowest layer in the Host volume that does not span both the Host and Controller. It provides 
logical links between multiple upper layer protocols (ATT, SDP, and SMP) to multiplex a variety of protocol operations between separate devices.

The L2CAP contains two units: a channel manager and a resource manager (Figure 2.8). The channel manager is responsible for the maintenance of the logical channels used within the L2CAP layer. The resource manager is responsible for managing the flow of data through the channels maintained by the channel manager. This is done by providing support for packet segmentation and reassembly as well as for error control and flow control.

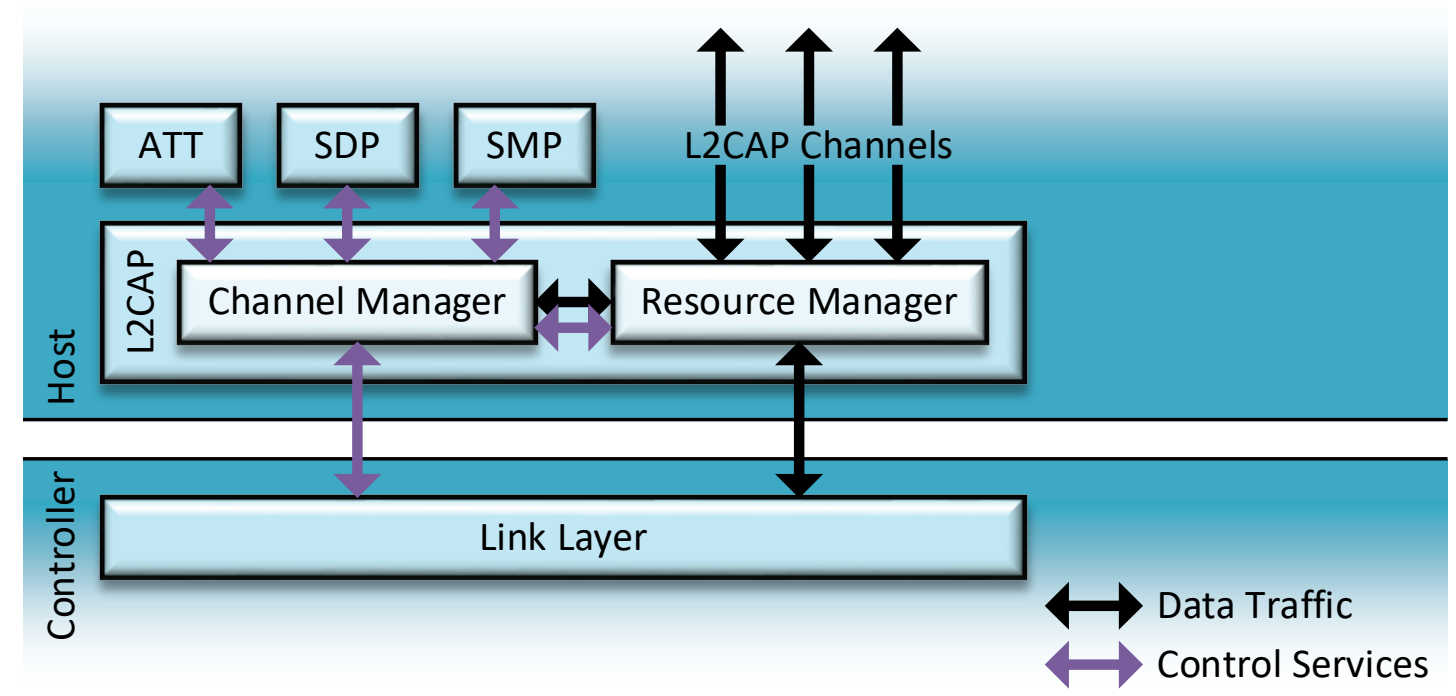

Figure 2.8. The structure of the L2CAP layer.

While important, both the $\mathrm{HCI}$ and L2CAP layers do not provide special functions that are targeted at the application described in this work. They have been included for the sake of completeness in this section of the work.

Attribute Protocol (ATT): An attribute is the fundamental unit of data used in BLE communication. Attributes are values which have a type, a handle, and permissions. The several types of attributes only have meaning in the context of the GATT profile, and are discussed in the following section. The attribute handle is a number used to access to identify a specific attribute within the GATT profile. The permissions associated with an attribute specify read/write access, encryption/security settings, and the enablement of notifications or indications. 
Notifications and indications are used to push new data to connected devices. A device with both notifications and indications disabled will not push data to paired devices. Notifications allow changes to attributes to be pushed to connected devices, but require no acknowledgement that the data was received. Indications push new data to connected devices and require acknowledgment packets to ensure the reliable delivery of the data.

There are two roles are specified at the ATT level: the client and server. The server is a device which locally stores the attribute data that is accessed by the client. Client and server roles are defined for individual procedures, so that the server in one procedure may not be the server for all procedures.

Six types of Protocol Data Units (PDU) are associated with the ATT protocol. Requests are transmitted by the client to request the server to provide information. Responses are the replies to these request packets sent from the server to the client. Commands are transmissions initiated by the client to cause the server to perform an action. Notifications and indications, described above, are data packets initiated by the server and sent to the client. Confirmations are packets sent from the client to the server when an indication packet was received.

Generic Attribute Profile (GATT): The GATT profile represents the hierarchical storage of device data that may be communicated between connected Bluetooth devices. The levels of this hierarchy are shown in Figure 2.9. The profile is composed of one or more services. Each service contains one or more characteristics. Each characteristic contains a value, properties, and may include one or more descriptors. Each service, characteristic, property, and descriptor is an attribute defined by the Attribute Protocol. The various types of attributes used in the GATT profile are identified using two-byte Universally Unique Identifiers (UUID). 


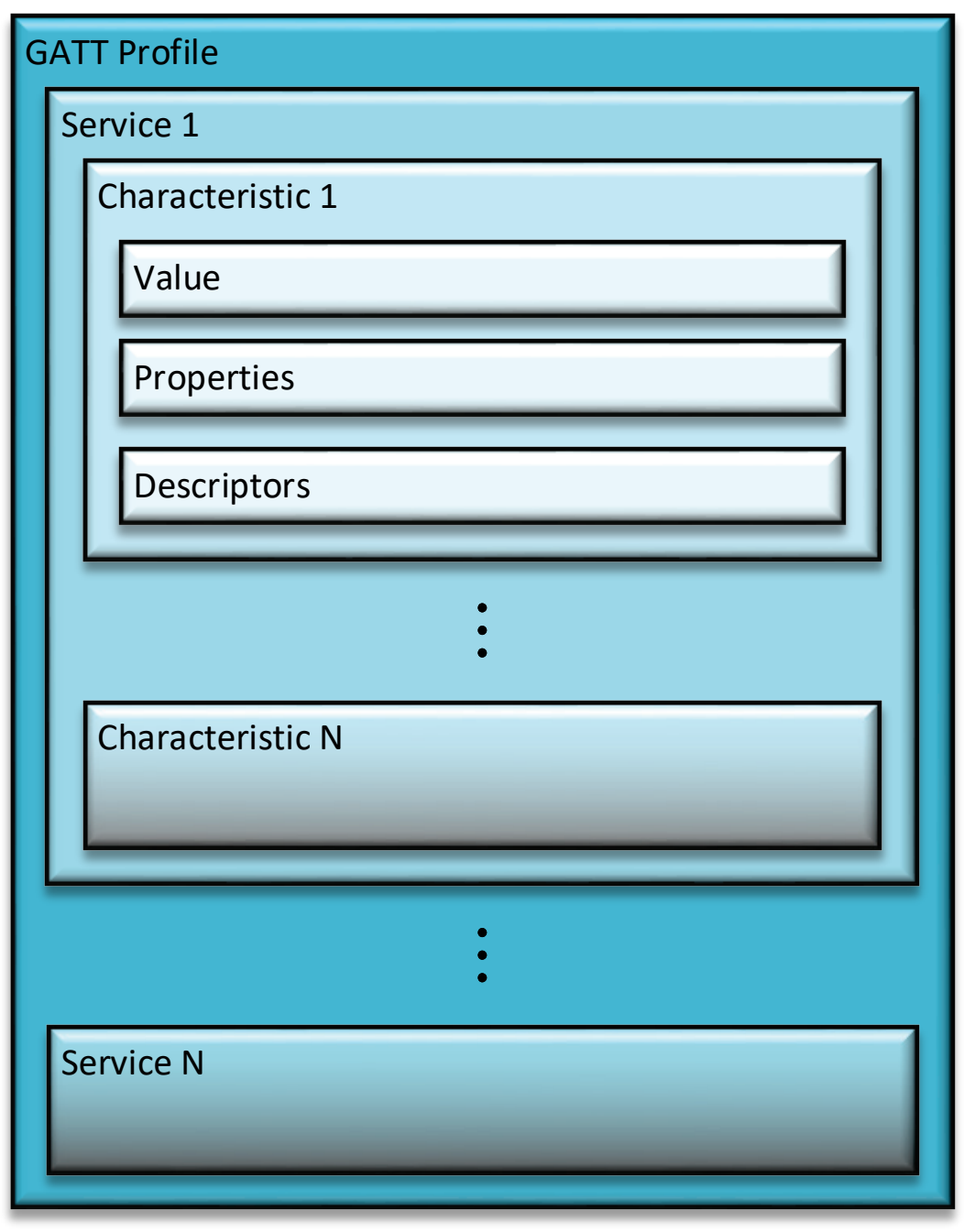

Figure 2.9. GATT structure.

A service is an attribute defined in the Bluetooth documentation as "any entity that can provide information, perform an action, or control a resource on behalf of another entity" [48]. There exist both primary and secondary services. Primary services represent the intended functionality of the device. Secondary services may be referenced by a primary service, but cannot be accessed directly. Services may also be included (or referenced) in other services that need the information from the referenced service.

A characteristic is an attribute within a service which contains data used or provided by the service. A single service may have characteristics. For example, a service that provides a temperature sensor reading may contain one characteristic containing a numerical value representing the temperature, and another representing the unit of 
measurement. A service to control an LED may have one Boolean characteristic to turn the light on and off, and another to control the brightness of the LED.

Further discussion on the use of the GATT profile in this application may be found in the following section (Section 2.2.2).

Service Discovery Protocol (SDP): The Service Discovery Protocol allows client devices to identify the services provided by nearby servers. Each device maintains a service record for each of its services. This service record contains a list of unique handles representing several service attributes which describe the service such as service name, service description, and service UUID. The SDP operates using request and response packets.

Clients may identify the services available in nearby clients by either a searching or browsing for services. By searching for services, the client can look for services whose attributes contain a particular value. This is done by searching for service attributes containing a desired UUID. Servers containing a service that matches the client's request will send a response to the client containing handles that the client can use to access the matching service. Alternatively, clients may browse all available services, and receive handles for each accessible service.

In the current work, the SDP is used significantly by the central device to differentiate peripherals that utilize the expected bioreactor service from other Bluetooth devices that may be within range. It is also used to identify different characteristics within that service so that $\mathrm{pH}$ measurements and temperature measurements are handled appropriately.

Security Manager Protocol (SMP): The Security Manager layer is responsible for creating secure connections between peer devices and ensuring that malevolent devices do not imitate the paired device. This is performed by the independent generation and distribution of keys to connected peers.

Before two peers have established a connection, the central device in the initiating state transmits a pairing request packet which provides the peripheral in the advertising state with information regarding authentication and security requirements. The peripheral replies with a pairing response packet which contains similar information. If this is successful, the central and then the peripheral will exchange pairing confirmation packets. 
After the confirmation packets have been received, both devices will then exchange random keys which can be used to encrypt the communication channel between the two devices.

Encryption may be an important concern to address in future implementations of this work. Biological data often has particular security needs that may be different from other applications. In this prototype, however, data has not been encrypted in order to save transmission time and, by extension, battery life.

Generic Access Profile (GAP): The GAP is the highest level of the Bluetooth stack. This profile represents how a device may relate to other devices in the network. This is defined using several roles, modes, and procedures. Roles are maintained throughout the lifetime of a device and dictate how one device interacts with devices with other roles. Two pairs of roles are used to define the operation of devices in the network: broadcaster/observer and central/peripheral.

The broadcaster and observer roles are used in broadcast networks in which only one node transmits data. This transmitter node is the broadcaster, and it is unable to receive information from other nodes. The receiver nodes are observers, and they are unable to transmit to other nodes in the network. Observers are only able to receive information, but not transmit. Broadcasters may be in the broadcast mode, or they may be in a periodic advertising mode that limits its visibility to the observer. The observer may be performing the observation procedure, or it may attempt to synchronize with a periodically advertising broadcaster. These roles have not been used in this work, but are used in certain applications.

The central and peripheral roles are used in point-to-point networks. Devices with the peripheral role do not operate in either the scanning or initiating states described in the link layer. They typically remain in the advertising state until a central device forms a connection with it. Peripherals always become the slave in point-to-point connections. Devices with the central role do not operate in the advertising state described in the link layer. Centrals scan for advertisers and initiate connections with them. Centrals always become the master in a point-to-point connection. In this work, all sensor nodes using the 
platform described below are peripheral devices. The central device is a USB hub that is operated using a computer.

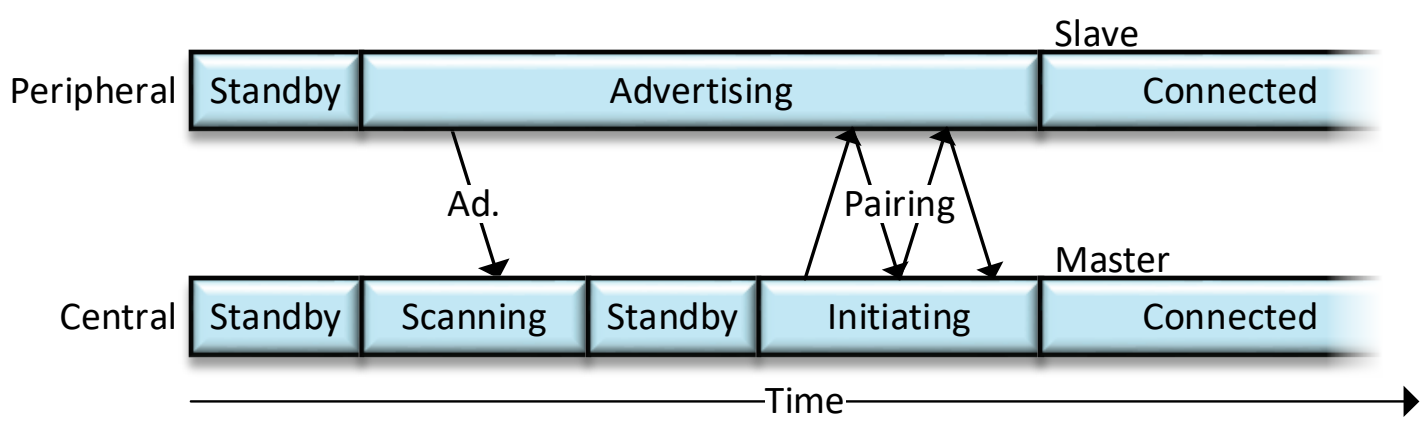

Figure 2.10. Connection procedure from startup.

In addition to the definition of these roles, the GAP also defines several modes and procedures that are used by Bluetooth devices. A procedure is an action or a series of actions initiated by one device in the network. A mode describes how a device responds to a procedure. Unlike roles, modes may change throughout the operation of the device. These modes and procedures relate to broadcast/observation, discovery, connection, and bonding.

The broadcast mode and observation procedure are used in broadcast network topologies. Devices with the broadcaster role remain in the broadcast mode, in which they periodically broadcast data without forming connections with other nodes. Observers continually perform the observation procedure in which they receive data from nearby broadcasters.

Three modes and three procedures are defined relating to node discovery. Peripheral devices may be in the non-discoverable mode, limited discoverable mode, or general discoverable mode. Non-discoverable peripherals cannot be discovered by any central devices. Peripherals in the limited discoverable mode may be discovered for a limited period of time after entering that mode. Peripherals in the general discoverable mode remain discoverable until changed to the non-discoverable mode. Central devices may perform limited discovery or general discovery procedures. The limited discovery procedure only discovers devices in the limited discoverable mode, while the general discovery procedure may find any device that is not in the non-discoverable mode. A name discovery procedure also exists, which may be used by either the central or peripheral to find the name of a particular device. 
Three modes and six procedures relate to the formation of connections between devices. Peripheral devices may be in the non-connectable, directed connectable, or undirected connectable modes. Devices in the non-connectable mode cannot establish connections with other devices. Broadcasters and observers are always in this mode. Peripheral devices in the directed connectable mode can form connections only with known peer devices, while undirected connectable mode allows for connection with any peer device. Central devices may initiate connections using the auto connection establishment procedure, general connection establishment procedure, selective connection establishment procedure, or direct connection establishment procedure. The auto connection establishment procedure allows the Controller (without direction from the Host) to autonomously form a connection with any device stored in a white list in the Controller. The general connection establishment procedure allows the Host to connect to any known peripheral. The selective connection establishment procedure allows the Host to connect to known peripherals limited by a set of parameters. The direct connection establishment procedure allows the Host to only a single known peripheral. Both the central and peripheral may initiate the connection parameter update procedure or the terminate connection procedure, which may force an update to the link layer parameters or sever the connection respectively.

Bonding modes and procedures relate to the formation and maintenance of secure connections. Both the central and peripheral may be in the non-bondable mode or the bondable mode, which determine whether or not the device can establish a secure connection. Both the central and peripheral may also initiate the bonding procedure to create a secure connection with a device in the bondable mode.

The device described in this work operates using a point-to-point topology. Before connections are established, the peripheral operates in the general discoverable and general connectable modes. This allows them to be discovered by and form connections with any appropriate nearby device. 


\subsubsection{GATT Profile}

The GATT profile used for this device contains all sensor platform characteristics in a single custom service. Five characteristics are used. Three of the five characteristics contain values for the $\mathrm{pH}$ measurement, temperature measurement, and voltage supply reading. Each of these have a length of 2 bytes, and require indication packets. The fourth characteristic provides remote control over the peripheral device by a central computer. This characteristic controls the sleep/wake state of the peripheral device and provides access to a remote reset function. The final characteristic is a contains no data, but is used to inform the central device that the peripheral provides $\mathrm{pH}$ data. The device name and default appearance, as well as manufacturer information, are also contained within the GATT profile. The profile used in one sensor device is shown in Figure 2.11. 

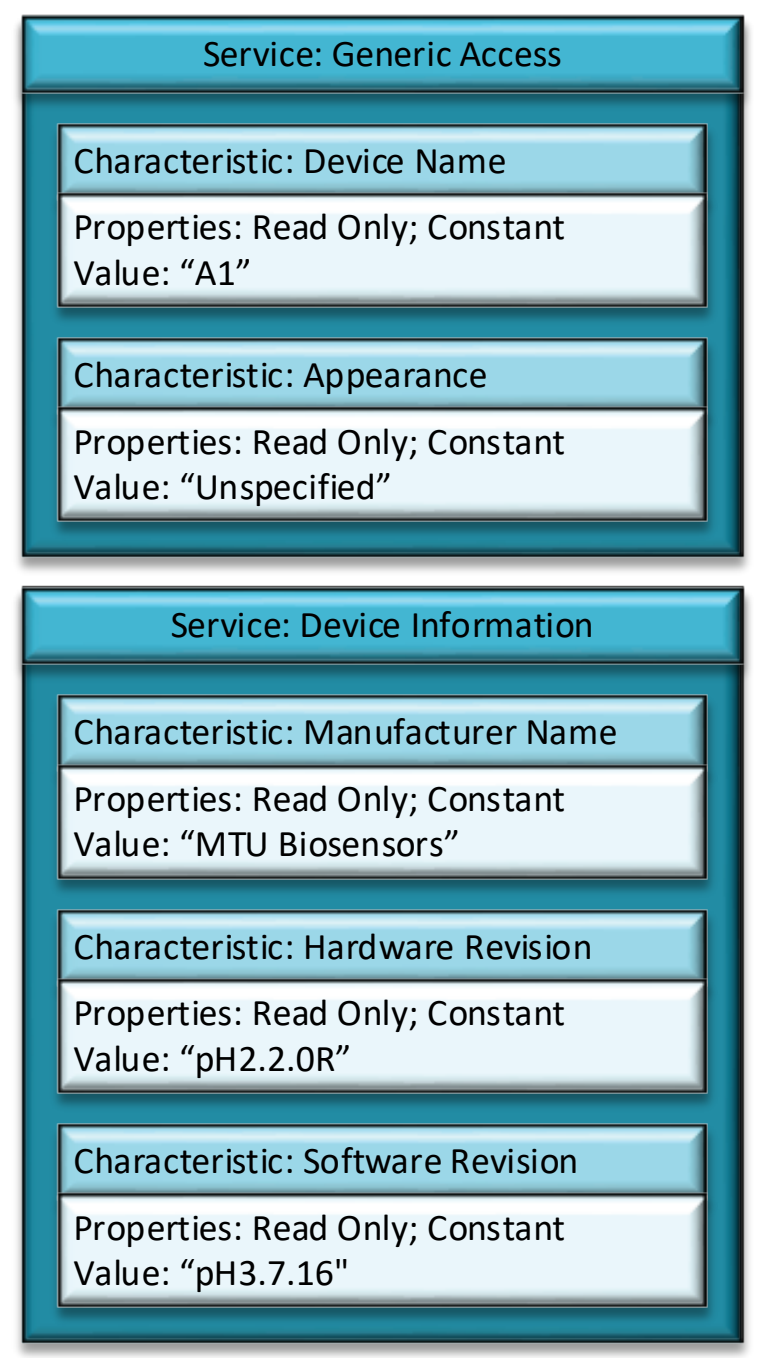

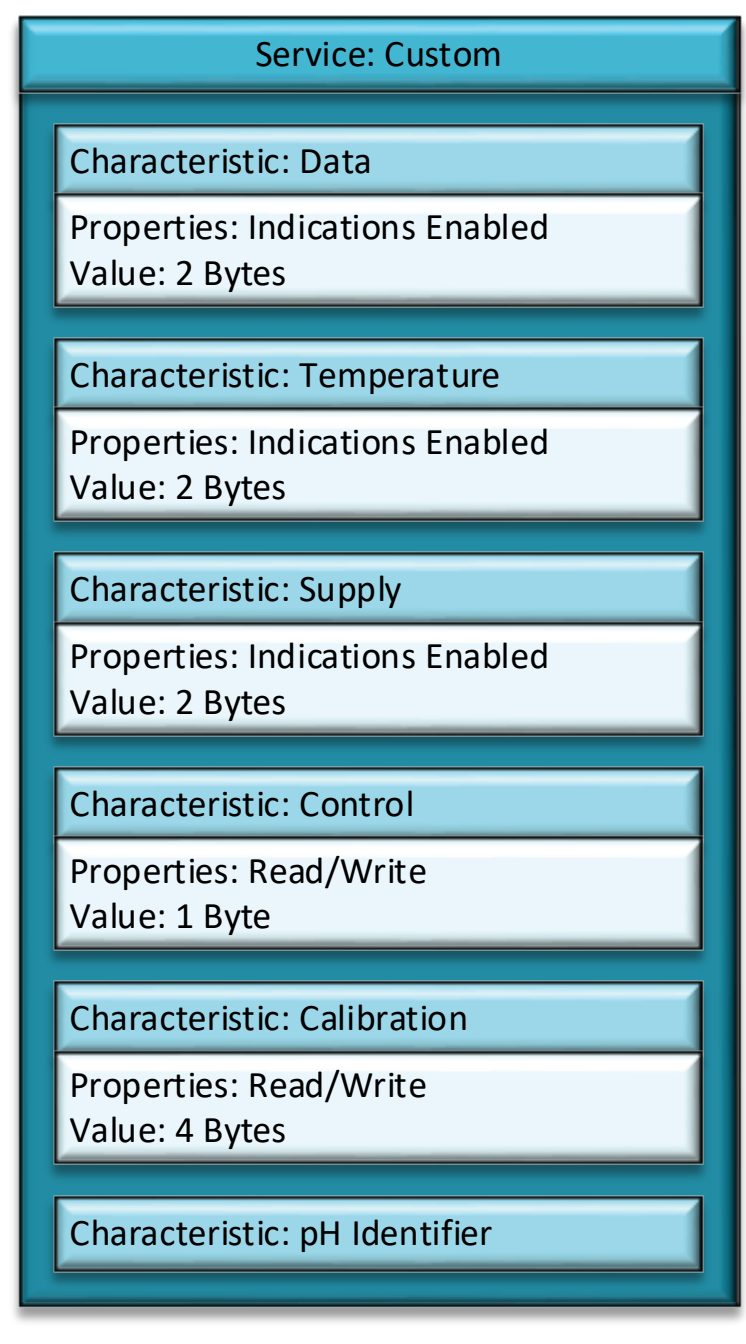

Figure 2.11. GATT profile of sensor A1.

Three services are used in this sensor platform. The first-the "Generic Access" service - contains a device name ("A1" in this case) and an appearance. The appearance contains information about what type of device this is, such as a thermometer or audio device. Since this sensor platform does not fit in any of the specified categories, its appearance is unspecified. The use of this service is required in Bluetooth devices, and provides convenient identification of each device when multiple devices are present.

The second service provides further information about the device. It identifies the manufacturer of the sensor, the hardware the device uses, and the software version number. This service provides invaluable information as both the sensor hardware and software 
undergo revision over time. Providing access to the revision details allows for remote differentiation between devices that have different functionality.

The third service is a unique service used by this sensor platform, and contains both data packets and accessible controls. The data characteristic contains the $\mathrm{pH}$ measurements transmitted by the sensor as a 16-bit signed integer. It does not specify that the data contains $\mathrm{pH}$ values in order to maintain interoperability between different versions of the software. The temperature and supply characteristics similarly contain 16-bit integer values for both temperature and the $1.25 \mathrm{~V}$ rail as measured by the ADC. Both are transmitted as integer $\mathrm{ADC}$ values, but are explicitly identified as containing temperature and supply values. These three are converted into the appropriate units by the software of the central device. Indications are enabled for each of these three packets so that any lost packets are retransmitted by the sensor. The control characteristic provides remote access to the device configuration. This characteristic is used to wake the device from sleep mode or return it to sleep mode, and to reset the device. These are read/write enabled so that they can be set by the client, as well as so the client can see if the sensor is currently asleep or awake. The calibration characteristic contains a 32-bit floating point value which may be read by the client to receive the stored calibration value of the sensor or written by the client to save a new calibration value. The $p H$ identifier characteristic contains no data or properties. It is used to identify that the data characteristic contains a $\mathrm{pH}$ value and should be interpreted accordingly.

\subsubsection{The BLE113}

The Silicon Labs BLE113 module contains the communication device, controller, and memory units shown in Figure 2.3. The controller is based on the TI CC2541 and provides $256 \mathrm{~KB}$ of flash memory. A small dipole antenna serves as the communication device. The BLE113 utilizes the Low-Energy protocols described in the Bluetooth 4.0 specifications. The entire module has a footprint of less than $16 \mathrm{~mm} \times 10 \mathrm{~mm}$ and a height of less than 2 $\mathrm{mm}$. 


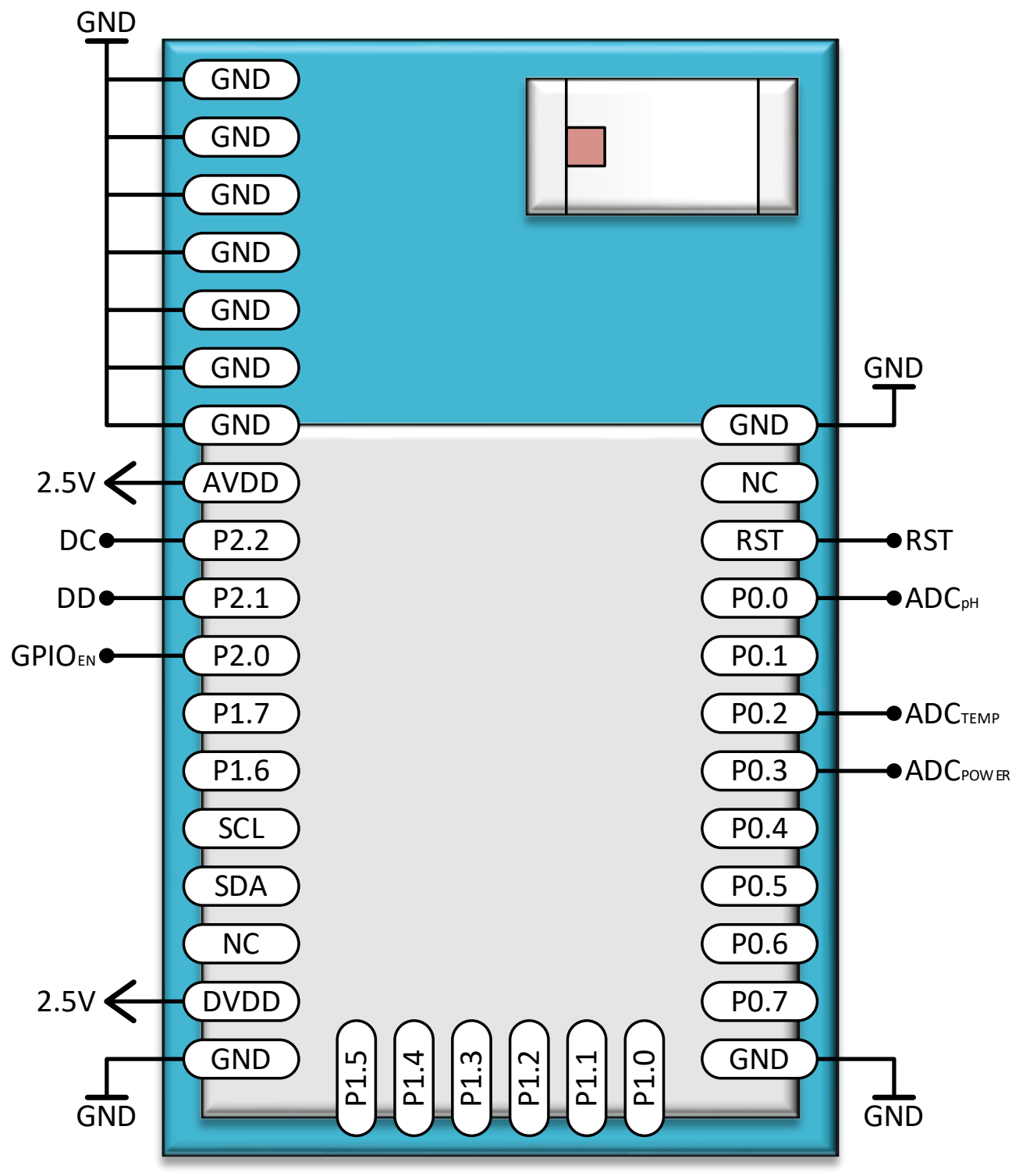

Figure 2.12. BLE113 connections.

The pins available on the BLE113 are shown in Figure 2.12. The microcontroller is powered by a $2.5 \mathrm{~V}$ supply. All eight pins on port 0 are available for use as ADCs with up to 12-bit resolution. Three of those pins are in use as ADCs in this sensor node: one to measure $\mathrm{pH}$, one to measure temperature, and one to monitor the voltage supplied by the 1.25 V rail (discussed in Section 2.5.5). One pin on port 2 is used as a GPIO to enable the secondary voltage regulator (discussed in Section 2.5.5). The remaining two pins on port 2 are used to program the microcontroller along with the reset pin. Programming may be 
performed using the Texas Instruments CC Debugger, which may connect to the programming pads using pogo pins.

A magnetic reed switch has been used to provide a remote emergency reset function. This reed switch is normal open and is located between the active-low reset pin of the BLE113 and the ground plane. Placing a magnet in the vicinity of the switch will cause an automatic reset of the device without requiring physical access to it.

\section{$2.3 \mathrm{pH}$ Sensor}

\subsubsection{Electrode Design}

Most commercially available $\mathrm{pH}$ sensors are either too large and too expensive to be used in this sensor application. Therefore, they cannot meet the specifications listed in Table 1.1. Instead of using these commercial options, custom sensing and reference electrodes were developed by our lab. The $\mathrm{pH}$ sensor consists of an antimony sensing electrode and a silver-silver chloride reference electrode [49]. This $\mathrm{pH}$ sensor configuration was chosen because it has been demonstrated to be stable, low cost, and easy to replicate.

The half-cell equations of the antimony and silver-silver chloride electrodes are shown in equations 2.1 and 2.2 respectively.

$$
\begin{gathered}
2 \mathrm{Sb}+3 \mathrm{H}_{2} \mathrm{O} \rightarrow \mathrm{Sb}_{2} \mathrm{O}_{3}+6 \mathrm{H}^{+}+6 e^{-} \\
\mathrm{AgCl}+e^{-} \rightarrow \mathrm{Ag}+\mathrm{Cl}^{-}
\end{gathered}
$$

The enclosure of the $\mathrm{pH}$ sensor (Figure 2.13) was 3D printed in two parts using a proprietary standard clear resin on the FormLabs Form 2 printer. The customized packaging shape allows for the reference electrode to fit neatly beside the sensor to minimize unused space in the sensor. The interior of the bottom of the reference electrode contains a screw-like texture to hold the agar salt-bridge in place and improve the lifetime of the sensor. Two 0.018 " holes were drilled in the packaging, one at the bottom of the body to allow for ion diffusion, and one in the cap for insertion of the silver-silver chloride reference electrode. This electrode was created by electroplating silver chloride onto a 20 $\mathrm{mm}$ silver wire, and glued in place using Loctite liquid super glue. $0.5 \mathrm{~mL}$ of agar gel filled the lower portion of the electrode, which features several ridges to help hold the gel in 
place. Once this agar gel had set, the remainder of the electrode body was filled with a $3 \mathrm{M}$ potassium chloride $(\mathrm{KCl})$ solution. The cap was then glued to the body of the reference electrode. This $\mathrm{pH}$ electrode packaging is $7 \mathrm{~mm} \times 13.5 \mathrm{~mm}$ x $23 \mathrm{~mm}$ in dimension. The small $\mathrm{pH}$ electrode size is critical to keep the whole sensor node within the size specification (see Section 2.6 for design and size of the sensor node).
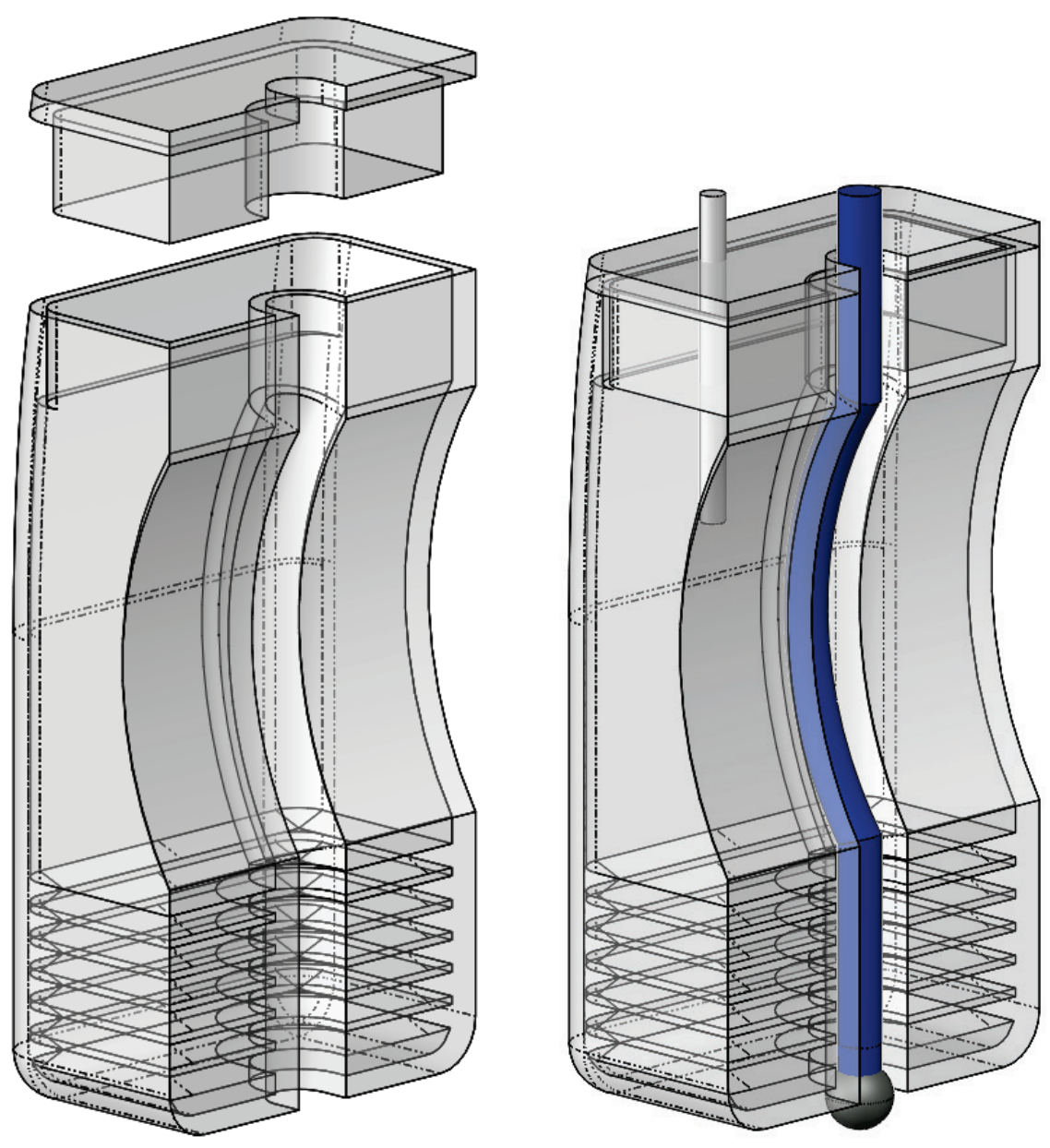

Figure 2.13. pH sensor design (left: exploded packaging).

The antimony sensing electrode was fabricated by allowing pure liquid antimony to harden on the end of a copper wire. Performix-brand plastic dip was used to cover any exposed wire. This electrode was then glued in place within a channel in the body of the reference electrode. 


\subsubsection{Signal Processing}

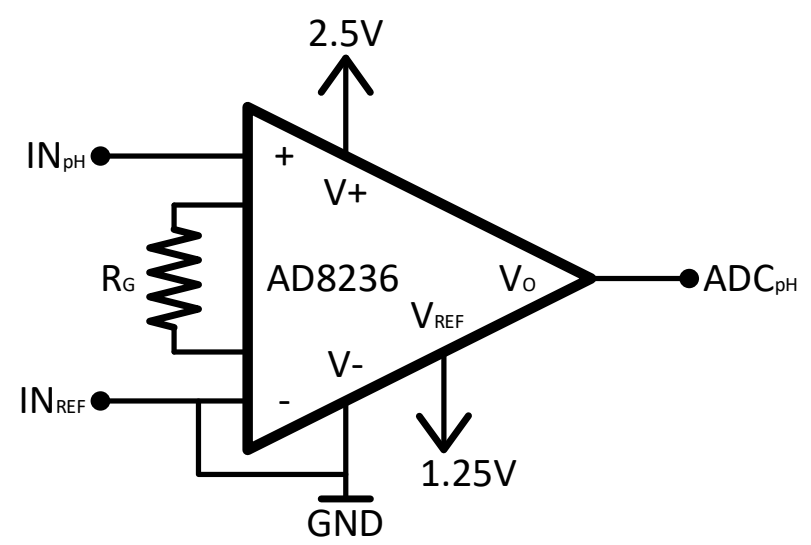

Figure 2.14. pH sensor amplification schematic.

The Analog Devices AD8236 instrumentation amplifier was used to increase the resolution of the $\mathrm{pH}$ measurements. The gain of the amplifier can be adjusted using resistor $R_{G}$. For this application, the gain of the system was set to 5 . The equation representing the output of the amplifier is shown in Equation 2.3 [50] where $G$ represents the gain of 5, $V^{+}$ and $V^{-}$represent the voltage at the two input terminals, and $V_{\text {ref }}$ equals $1.25 \mathrm{~V}$ and is supplied to the reference pin.

$$
V_{o}=G\left(V^{+}-V^{-}\right)+V_{r e f}
$$

\subsection{Temperature Sensor}

Temperature monitoring of the cell culture is performed using a low-power temperature sensor. The Texas Instruments LMT89 temperature sensor was selected for this purpose due to its small footprint and accuracy of $\pm 1.5^{\circ} \mathrm{C}$ or minimum accuracy of $\pm 2.5^{\circ} \mathrm{C}$ at the temperature extremes of $-55^{\circ} \mathrm{C}$ and $130^{\circ} \mathrm{C}$. The temperature sensor's connections are shown in Figure 2.15. 


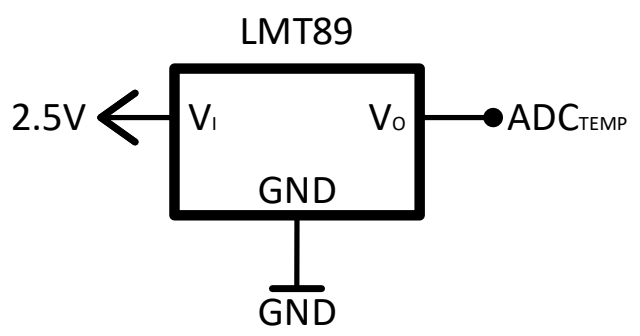

Figure 2.15. Temperature sensor schematic.

The temperature sensor is supplied by a $2.5 \mathrm{~V}$ supply rail. The temperature sensor can be disabled during sleep mode by disabling this voltage supply. The output of the temperature sensor is measured by one of the ADCs present on the BLE113. The temperature is calculated from the output voltage $V_{o}$ using equation 2.4 [51].

$$
T=-1481.96+\sqrt{2.1962 \times 10^{6}+\frac{1.8639-V_{o}}{3.88 \times 10^{-6}}}
$$

Using the 12-bit ADC, this temperature sensor may detect changes in temperature with a resolution of $0.048^{\circ} \mathrm{C}$. The accuracy of the sensor will be limited somewhat by its enclosure in the biocompatible packaging, and therefore its separation from the cell culture substrate.

\subsection{Power Supply}

Since the sensor will be submerged in liquid, it was hermetically sealed (see Section 2.6) to prevent exposure of the electronic components to the growth medium. Therefore, when used as a disposable sensor for short-term monitoring, the sensor will be equipped with a larger-capacity battery $(>500 \mathrm{mAh})$ to extend battery life. In contrast, for long term monitoring or when footprint is an issue, a power supply system that can be powered or recharged without opening the packaging will be used. Figure 2.16 demonstrates the three types of powering schemes for this sensor. 


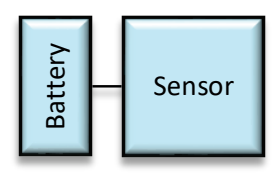

A) Non-Rechargeable

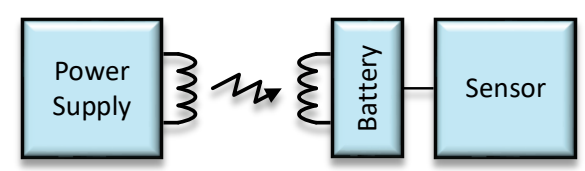

B) Wirelessly Rechargeable

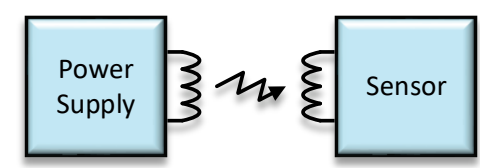

C) Wirelessly Powered

Figure 2.16. Sensor powering schemes.

A rechargeable system is typically based on inductive charging. An inductive coil presented with an AC signal will generate a magnetic field. If a second inductive coil is exposed to this field, a current reflecting the original AC signal will occur in this receiver coil. Systems using inductive charging typically have a very limited range of only a few millimeters and even less freedom in the $x-y$ directions. An inductive charging system that can significantly improve the charge transfer efficiency is to operate in the resonance mode, in which power is transmitted only at the resonant peak.

In this work, a sensor with a rechargeable system was developed to demonstrate its capability. A non-rechargeable sensor will be identical with the rechargeable version, except directly powering the circuits from the battery rather than the rechargeable circuit. The remote powered system, although a viable potential, is not considered in this work as there are still concerns of power loss when the charger unit is misaligned, thus causing loss of sensor data during operation.

\subsubsection{Battery Selection}

The power supply will be both internal and rechargeable, therefore a rechargeable battery must be used. Two most common batteries available on the market today are based on lithium ion (Li-Ion) and lithium polymer (LiPo) technologies. Both types of batteries are functionally similar, only Li-Ion use a liquid electrolyte while LiPo use a polymer electrolyte.

Both types of batteries are available in different sizes with differing capacities. Typically, battery packs are custom made to maximize the charge capacity for the available space. Several companies offer multiple sizing options for much lower costs. Alternatively, Li-Ion and LiPo batteries can be made into the shapes of conventional battery cell, like watch batteries. 
The battery used in this work is a rechargeable lithium ion battery with a CR2032 form factor developed by Illinois Capacitor (model RJD2032C1). This battery can supply a maximum of $3.7 \mathrm{~V}$ at up to $170 \mathrm{~mA}$, though the standard discharge current is approximately $17 \mathrm{~mA}$. The maximum capacity of this battery is $85 \mathrm{mAh}$.

\subsubsection{Wireless Battery Charging}

Several systems have been used to transfer power from one device to another without the use of wires. Long range wireless power transfer systems usually utilize microwaves or lasers to send energy over many kilometers. Short range wireless power transfer is often performed via magnetic induction. In magnetic induction, an AC power source is supplied to a primary inductor coil (the transmitter coil) which generates a localized magnetic field. If a secondary inductor coil (the receiver coil) is placed in the range of this magnetic field, that field will generate an alternating electric current in the coil. This current can then be used to charge a battery and power a wireless sensor like the one described in this work. A schematic diagram of a wireless battery charging circuit using magnetic induction is shown in Figure 2.17.

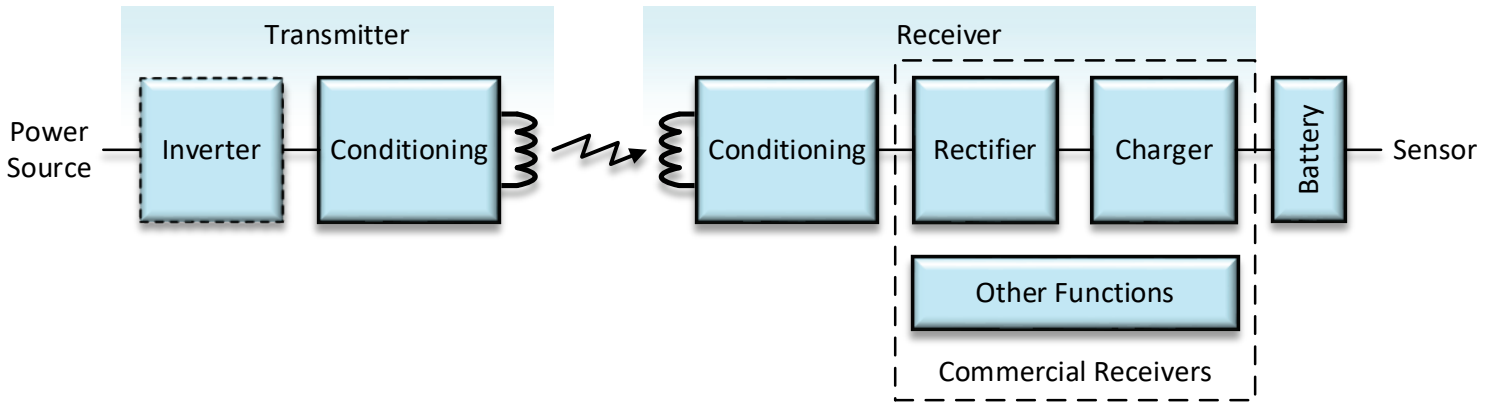

Figure 2.17. Wireless charging schematic.

Magnetic induction requires an alternating current source to drive the magnetic field. Many commercially available transmitters are supplied using a DC power source, often by means of a USB cable. This DC source is then converted to an AC signal at some desired frequency using a power inverter.

Before the transmitter coil and after the receiver coil is usually some sort of conditioning circuit. The purpose of these conditioning circuit is to maximize the efficiency of power transfer. This conditioning circuit usually contains an array of capacitors to tune 
the two coils by matching the impedances. A design that tunes the coils very closely so that they resonate at the same frequency as the signal as the signal is often said to be operating in resonance mode. This resonance mode can allow power transmission at significantly greater distances between the two coils, as well as allow spatial freedom in other directions so that the receiver can still receive power when off center or at an angle.

An AC signal will be the output of the conditioning block of the receiver after this procedure. A rectifier circuit is used to convert this AC signal back to a DC signal. Most rectification methods will result in some amount of ripple voltage and current, not a perfect DC signal. It is often helpful to pair the rectifier with some degree of voltage regulation so that the signal maintains the desired voltage and current as closely as possible regardless of environmental factors.

While it is possible to charge a battery using a constant voltage and current supplied by a regulator, different types of batteries can be charged more efficiently using different methods. For example, Li-Ion batteries use the constant current/constant voltage (CCCV) method shown in Figure 2.18. During the first stage of charging, a constant current is supplied to the battery, typically somewhere in the range of 0.5 to 1 times the maximum charge of the battery. When the voltage of the battery reaches a nominal max voltage described by the manufacturer, the charger switches to supplying a constant voltage as the current slowly drops.

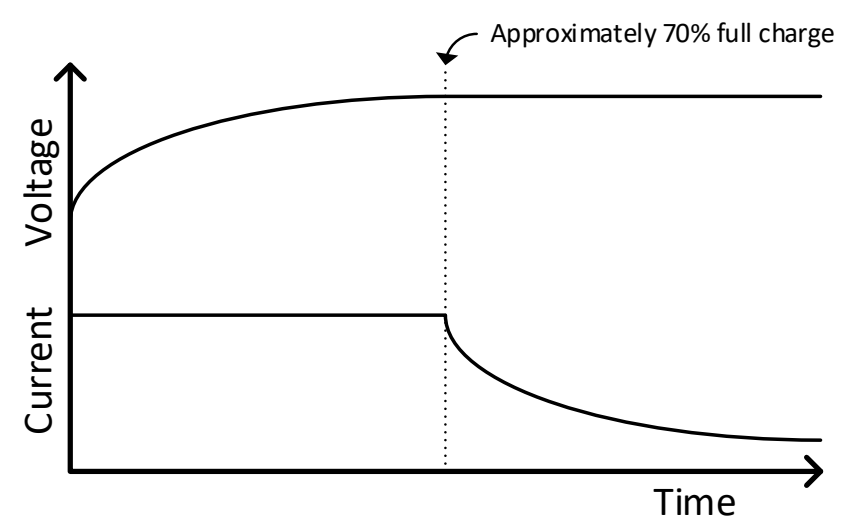

Figure 2.18. Lithium-ion battery charging curve.

Multiple commercial wireless charging standards exist to ensure compatibility with other devices. One example is the Qi standard officiated by the Wireless Power Consortium 
(WPC). The Qi standard includes other safety considerations not present in the above model. Wireless power transfer must stop if an object is detected between the transmitter and receiver. Communication protocols between the transmitter and receiver are present before and during power transfer. While the newest standards (WPC 1.2) have opened the door for resonant charging, the Qi standard has traditionally utilized inductive charging methods. One competitor to the Qi standard is Rezence, which is moderated by the Alliance for Wireless Power (A4WP). They have incorporated resonant charging characteristics into their designs, allowing for greater versatility.

\subsubsection{Wireless Power Receiver Design}

In order to minimize the space of the device, a two-layer "sandwich" board design has been used in which there are circuit boards both on top and bottom of a rechargeable CR2032 battery. One board contains the microcontroller and signal conditioning hardware, while the other contains the circuitry for the wireless power receiver.

In the current design, we have opted for a commercially available chip to handle the wireless power transfer. The Texas Instruments bq51050b is a WPC 1.1 compliant integrated circuit that requires only minimal external hardware to function, and serves as both the rectifier and battery charger in the receiver circuit. By using the WPC 1.1 standard, we are able to use off-the-shelf phone chargers as the transmitter. 


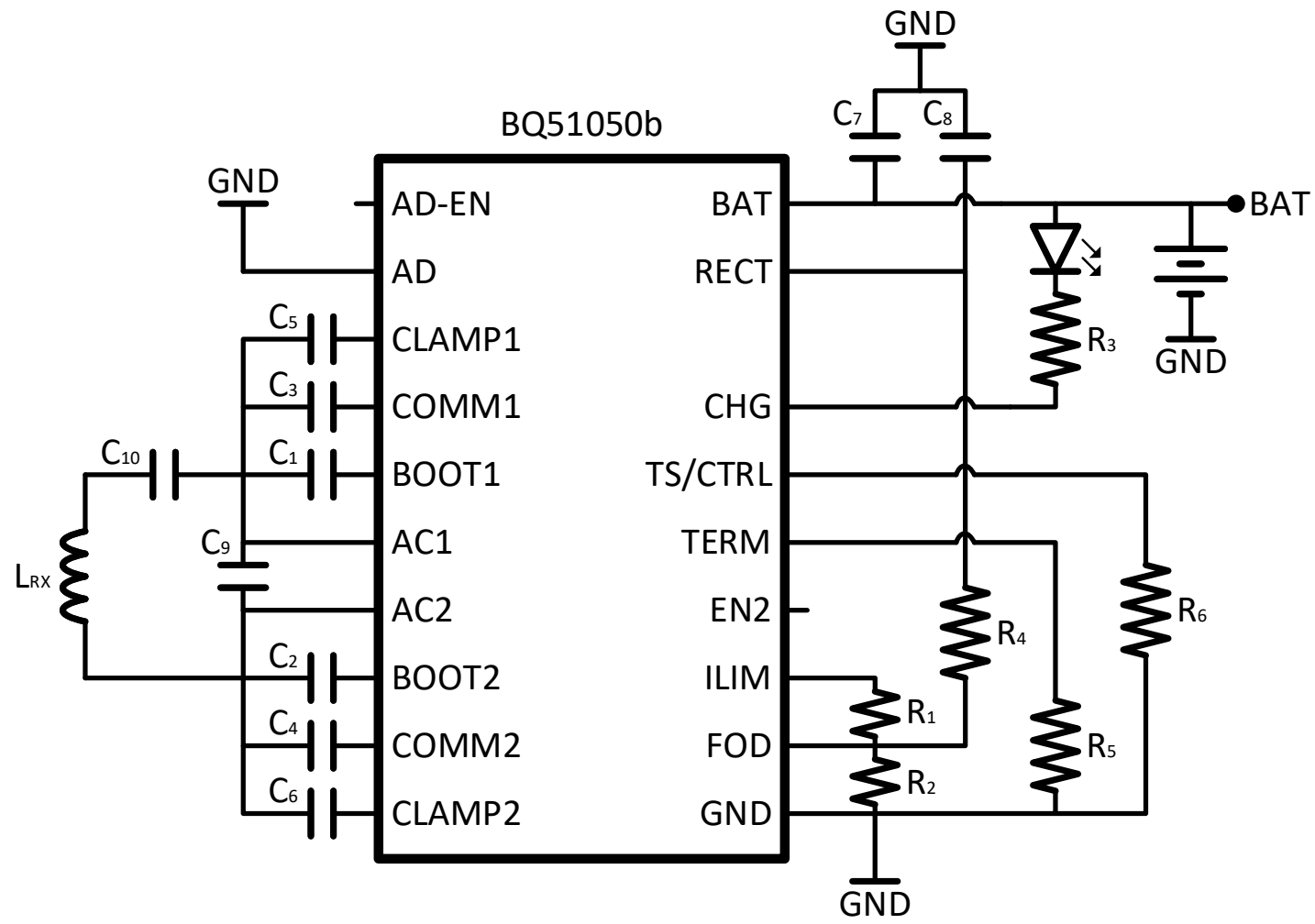

Figure 2.19. Wireless power receiver schematic.

The receiver coil being used is the Wurth 760308101214. It is a one-layer, $26 \mu \mathrm{H}$ inductor. This coil was chosen for two reasons. It has a diameter of $19 \mathrm{~mm}$, which fits nicely with a battery diameter of $20 \mathrm{~mm}$. It also has a relatively high inductance for coils of its size. The receiver coil is tuned using capacitors both in series and in parallel with it. Several other capacitors are also connected between the coil and the IC to perform several other functions.

There are several key features offered by this chip. An LED is connected between the positive terminal of the battery and the $\mathrm{CHG}$ pin to show when charging occurs. Several resistors have been used to set the battery charging current, to enable foreign object detection, and to set when charging should stop. There also exists a temperature sensing function of the chip that stops charging if the system gets too hot. This temperature sensing functionality will be of great use in later versions of the sensor, but it is not currently being utilized in this design due to manufacturing problems. 
Tuning the inductance of the coil is performed using capacitors $\mathrm{C}_{9}$ and $\mathrm{C}_{10}$. The distance between the transmitter and receiver coil is tuned using capacitors $\mathrm{C}_{3}$ and $\mathrm{C}_{4}$. Shutoff current is specified using resistors $\mathrm{R}_{1}, \mathrm{R}_{2}$, and $\mathrm{R}_{5}$.

\subsubsection{Wireless Power Transmitter Compatibility}

The current wireless power transfer system present on this sensor node is designed to be WPC 1.2 compatible. Therefore, any WPC 1.1 or later transmitter should be compatible with this sensor node. Many such transmitters are commercially available. In our lab, we have used a Samsung WPC 1.1 transmitter pad designed for charging phones. The range between the transmitter and receiver are limited by the small receiver coil implemented in this sensor design.

\subsubsection{Power Regulation}

Batteries do not supply a constant voltage at all times. As a battery discharges, the voltage it supplies will drop until the supply voltage is too low to continue to provide the needed power. This variation in voltage supplied to the microcontroller will increase the resolution of the ADCs and thereby reduce the accuracy of the sensor as a whole. Fluctuations in voltage supplied to other components, such as the temperature sensor, will also diminish sensor accuracy. It is therefore important to implement voltage regulation in a battery-powered sensor to prevent fluctuations in the battery voltage to affect the voltage supplied to the sensor node.

Two voltage regulators have been implemented in this sensor node. The first regulator is the Microchip MCP1700 low dropout voltage regulator (Figure 2.20). The battery does not directly supply power to any part of the sensor board. Instead, a stable $2.5 \mathrm{~V}$ rail is supplied by the primary voltage regulator to the BLE113 and to the secondary voltage regulator. 


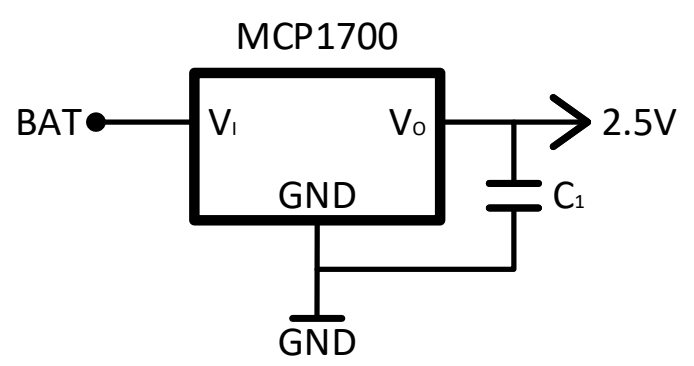

Figure 2.20. Primary voltage regulator.

The secondary voltage regulator features the Texas Instruments TLV711 (Figure 2.21). This regulator is used to power other components of the sensor node that may be disabled during sleep mode to save power. It also provides a $1.25 \mathrm{~V}$ rail in addition to a $2.5 \mathrm{~V}$ rail which is used to provide the reference voltage for the $\mathrm{pH}$ amplifier (Section 2.3.2). Both enable pins, used to enable the two voltage rails, are connected to the same GPIO pin on the BLE113 so that both supply rails may be turned on or off at the same time.

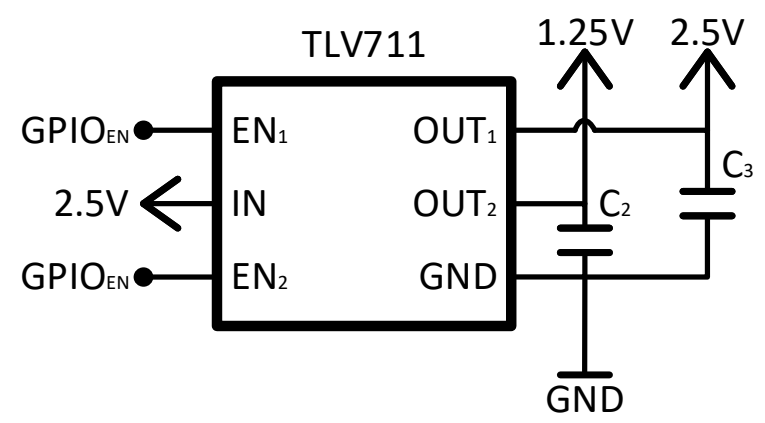

Figure 2.21. Secondary voltage regulator.

\subsection{Enclosure}

\subsubsection{Construction}

Four separate components (Figure 2.22) were 3D printed for the packaging of the sensor. These were created using the FormLabs Form 2 3D printer using their proprietary Standard Clear resin (RS-F2-GPCL-04). Two of these components were used to house the reference electrode, and were discussed in Section 2.3.1. The remaining two components contained the other components of the sensor platform. 


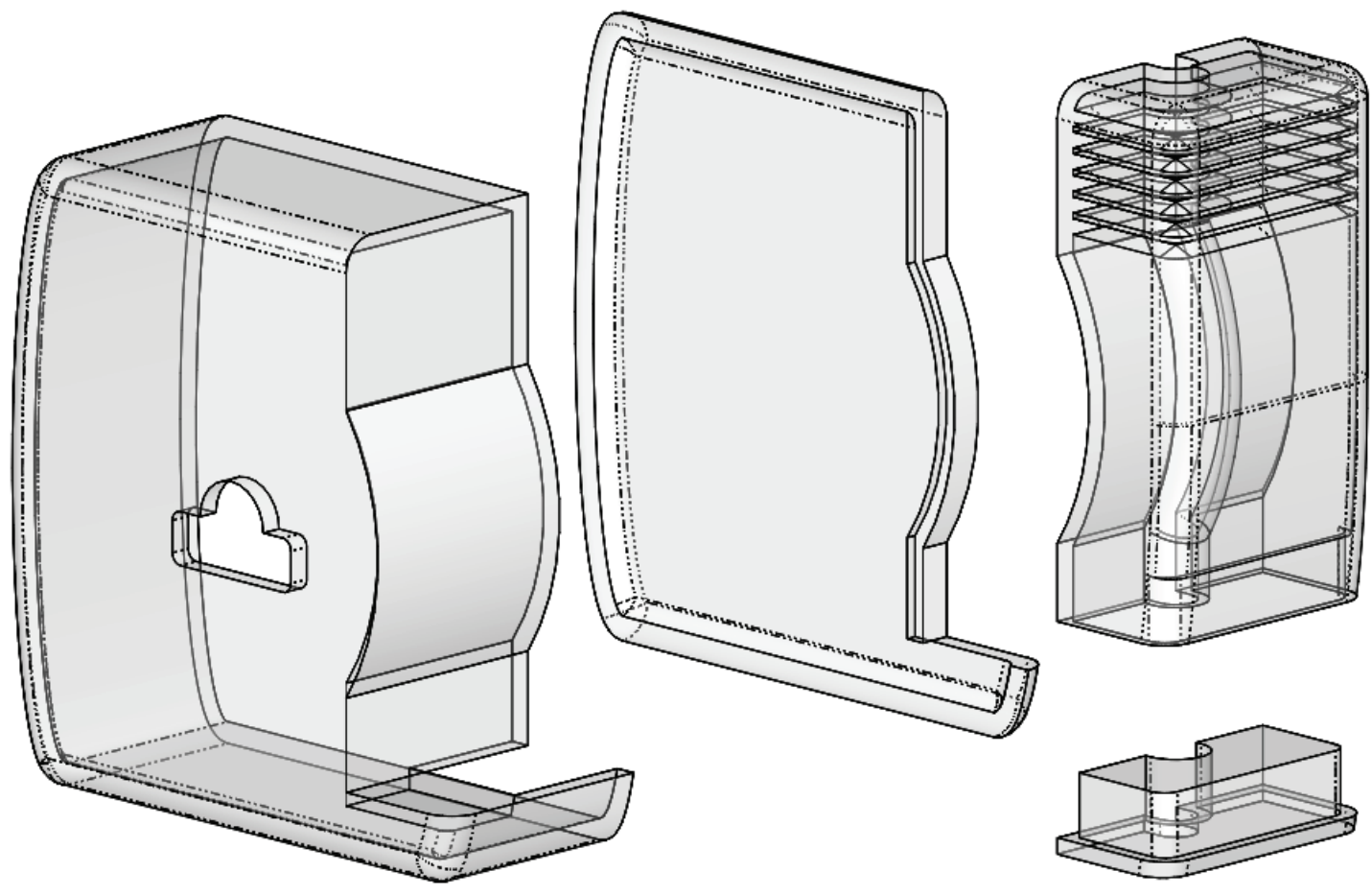

Figure 2.22. 3D printed packaging (exploded view).

A $20 \mathrm{~mm}$ diameter silicone suction cup was inserted into the face of the sensor packaging, and Loctite liquid super glue was used to plug the opening. Once the sensor platform was installed inside the enclosure, the front and back of the sensor packaging were glued together. The completed $\mathrm{pH}$ sensor was then also glued into place. The finished packaging is shown in Figure 2.23. The overall dimensions of the packaging for this sensor are less than $30 \mathrm{~mm} \times 30 \mathrm{~mm} \times 17.5 \mathrm{~mm}$ (13.5 mm without the suction cup). This allows it to be used in benchtop bioreactors such as the $500 \mathrm{~mL}$ Wheaton ${ }^{\circledR}$ Celstir Complete. 

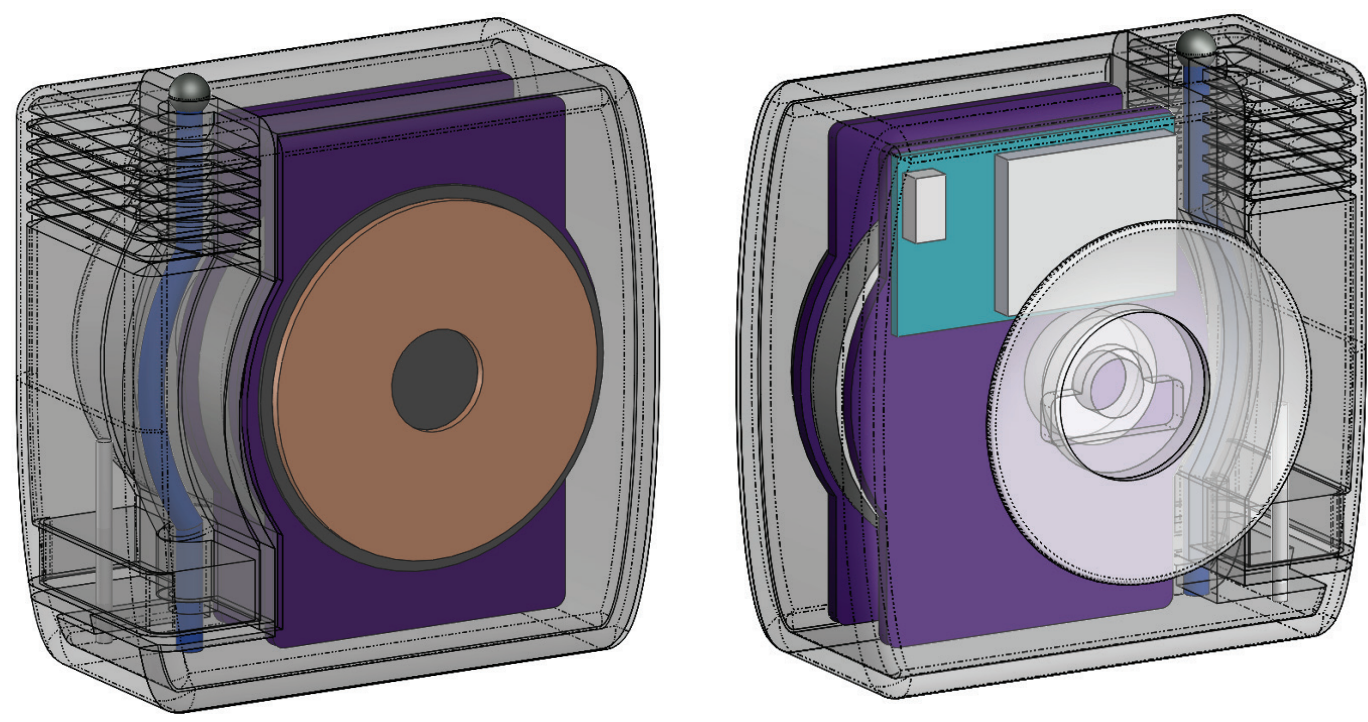

Figure 2.23. Sensor enclosure design.

\subsubsection{Biocompatibility and Future Changes}

Due to the limited project scope in creating a proof-of-concept prototype, the packaging used in this thesis has not been designed to meet biocompatibility standards. No biological matter will be used at this stage, so biocompatibility is not necessary, and more affordable materials may be used. However, similar techniques may be used in future designs. FormLabs offers a class 1 biocompatible resin aimed at applications in dentistry which may be used. Several biocompatible alternatives exist for adhesives as well. Sterilization of the sensor platform may be performed using ethylene oxide. It will also be necessary to perform a leeching test on the exposed tantalum electrode to determine its biocompatibility. Such a test is beyond the scope of this work, but it will be performed prior to testing of the material with biological matter.

\subsection{Operation of the Wireless Sensor}

When the peripheral device is powered on, it immediately begins broadcasting two advertising packets. One packet contains the name of the device, and the second contains a list of services offered. The central device, typically a user-operated computer, will provide a list of discovered devices containing the correct services and list them to the user by name. The user may then select the desired device and begin the connection process. 
Once the peripheral device has formed a link with the central device, it will stop advertising. By default, the peripheral device is in a sleep state and does not measure or transmit data. The only communication between the devices is a brief, periodic communication to maintain the link between the two.

Changing between the sleep and wake state is initiated by the central device. A packet with a one-byte payload called the control packet is used to change the sleep state, as well as to remotely reset the device when necessary. When the peripheral receives a control packet telling it to be in the wake state, it will turn on the secondary voltage regulator and begin two continuous timers, $\mathrm{T} 0$ for $\mathrm{pH}$ measurements and $\mathrm{T} 1$ for temperature and voltage supply measurements. When each timer expires, the appropriate ADCs are read.

When the ADC reading is complete, the data will be transmitted to the central device using separate packets for $\mathrm{pH}$, temperature, and voltage supply. Each packet requires the central device to confirm that it received the data before the next packet will be sent. In Bluetooth, these confirmations are known as indications. If an indication is not received within a certain window, the packet will be retransmitted. To manage which packets have been indicated and which have not in the peripheral device, since it is possible for all three packets to be ready simultaneously, three flags exist to mark that there is either $\mathrm{pH}$, temperature, or voltage supply data that is ready to be transmitted, but has not yet been indicated. When an indication is received, other pending flags are checked, and the next pending packet will be sent.

When the connection between the two devices is severed, either by command of the central at the behest of the user or due to loss of connection for some other reason, the peripheral device will immediately return to its advertising state.

A calibration function is also offered on this device. A calibration value may be transmitted to the sensor to be stored in local flash memory, which will remain when the device loses power. The sensor can then, upon request transmit this stored value to a connected device to acquire the appropriate $\mathrm{pH}$ conversion function. 


\subsection{Access Point Software}

To receive data from the sensors, an application was created using the $\mathrm{C} \#$ programming language. The wireless transceiver that acts as the central device is the BlueGiga BLED112 (Figure 2.24), a USB hub that interfaces with other BlueGiga BLE devices.

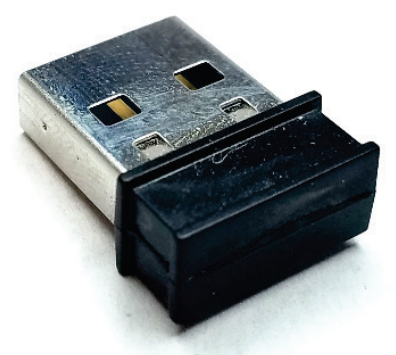

Figure 2.24. The BLED112.

Before the access point software can communicate with the sensor platform, a serial communication port must be opened to provide access to the BLED112. This is shown in Figure 2.25. Available ports are listed in a drop-down menu, which may be refreshed using the Refresh button. Once the desired port is selected, the Attach button will open communication with that port. 


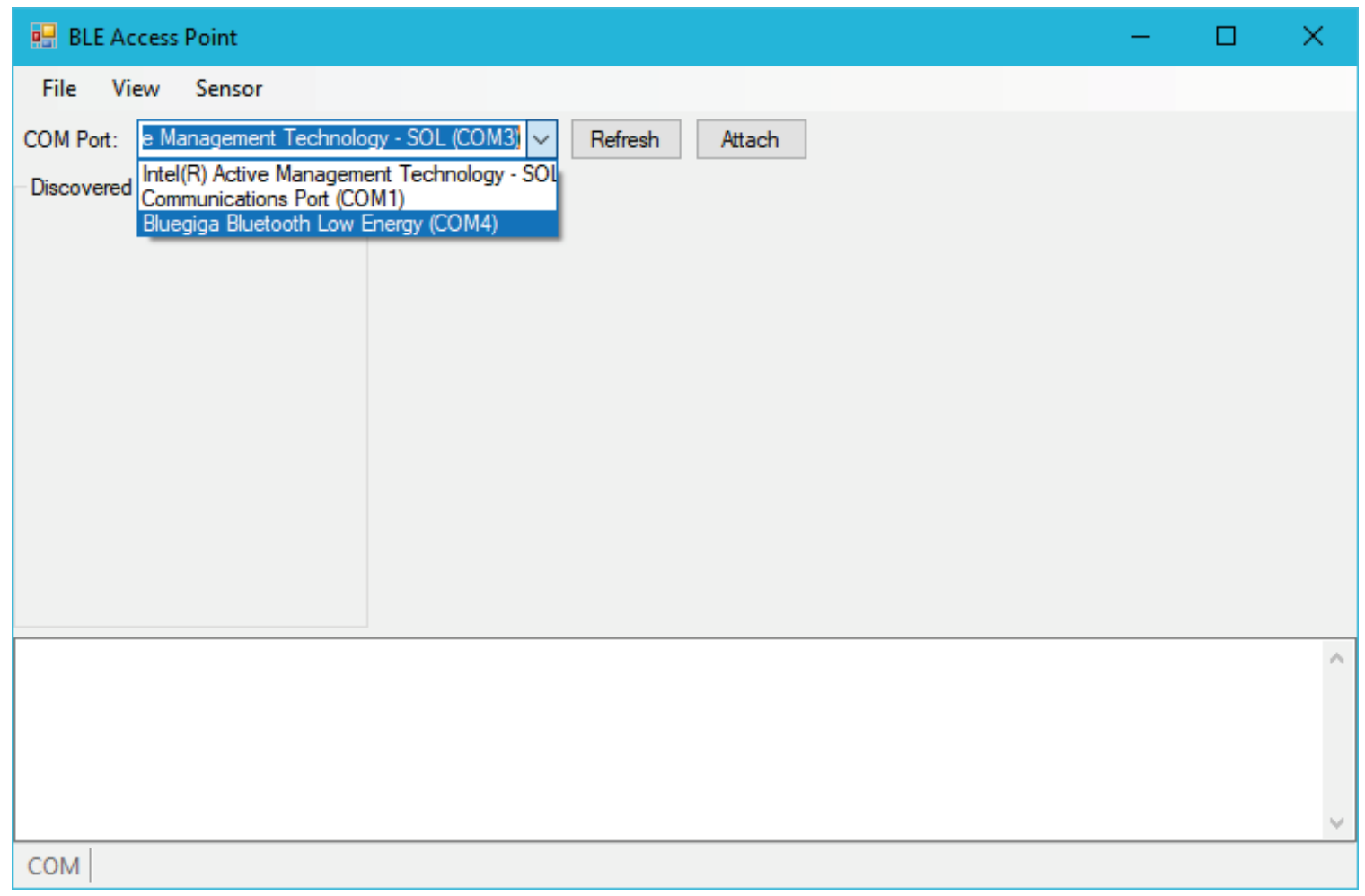

Figure 2.25. Connecting to the BLED112.

Once communication is opened with the BLED112, the software begins the scanning process. As advertisement packets from nearby devices are received, the list of services they offer are compared with a list of services that the software is compatible with. Any devices that contain the desired services are listed in the Discovered Devices panel (Figure 2.26). Each entry in the panel contains both the name and address of the discovered device. 


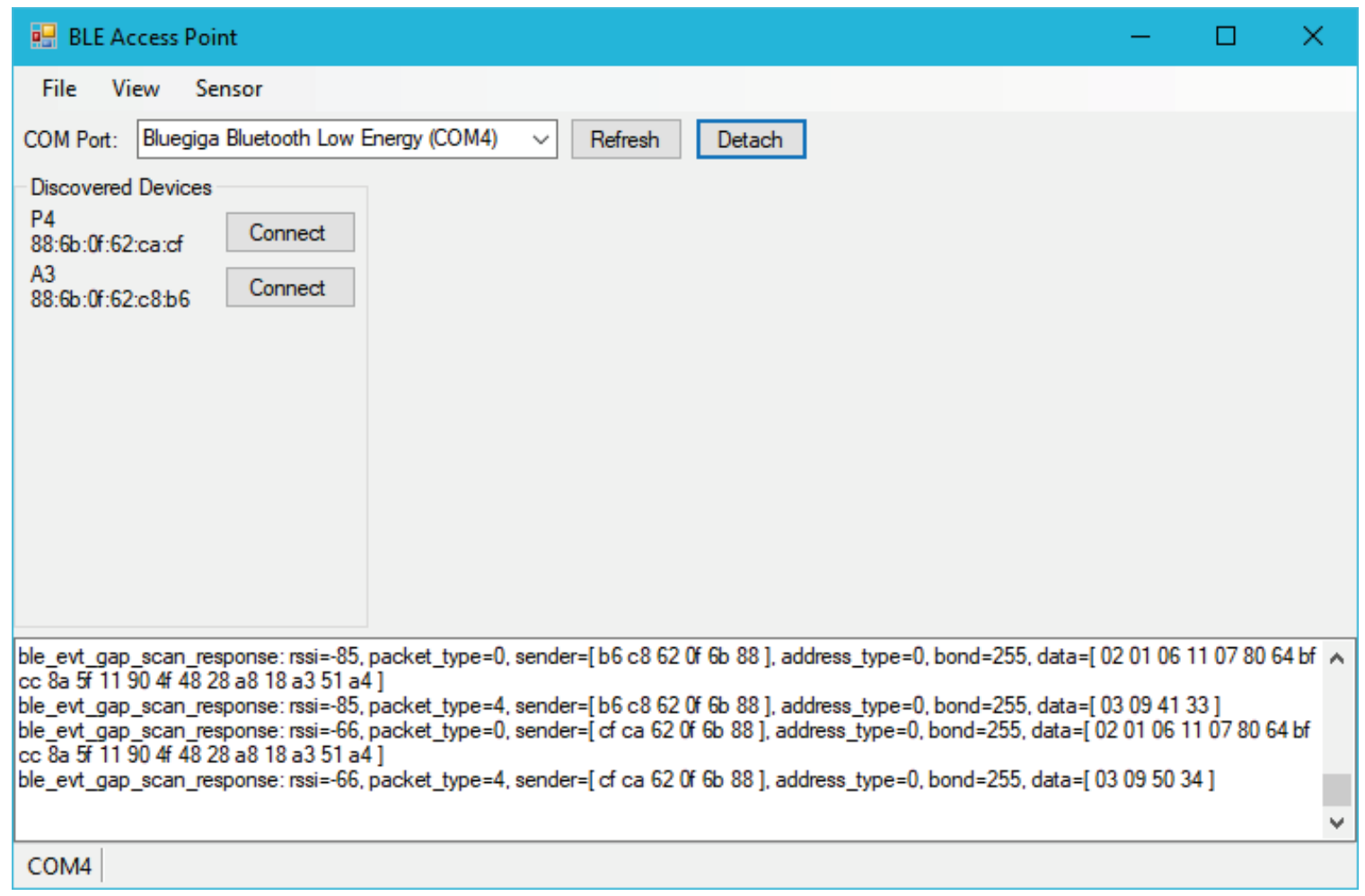

Figure 2.26. Listing of discovered devices.

The Connect button adjacent to each discovered device begins the connection process with that device. This process involves not establishing a connection with the peripheral device, but also getting information on the handles, or locations, of each characteristic they maintain, and enabling any notifications or indications that are offered by the peripheral device. A table is displayed in the interface once the peripheral is fully connected. In Figure 2.27, the connection was formed to a device in sleep mode. When the device is in sleep mode, it is not transmitting data to the central receiver, so no data is shown in the table. 


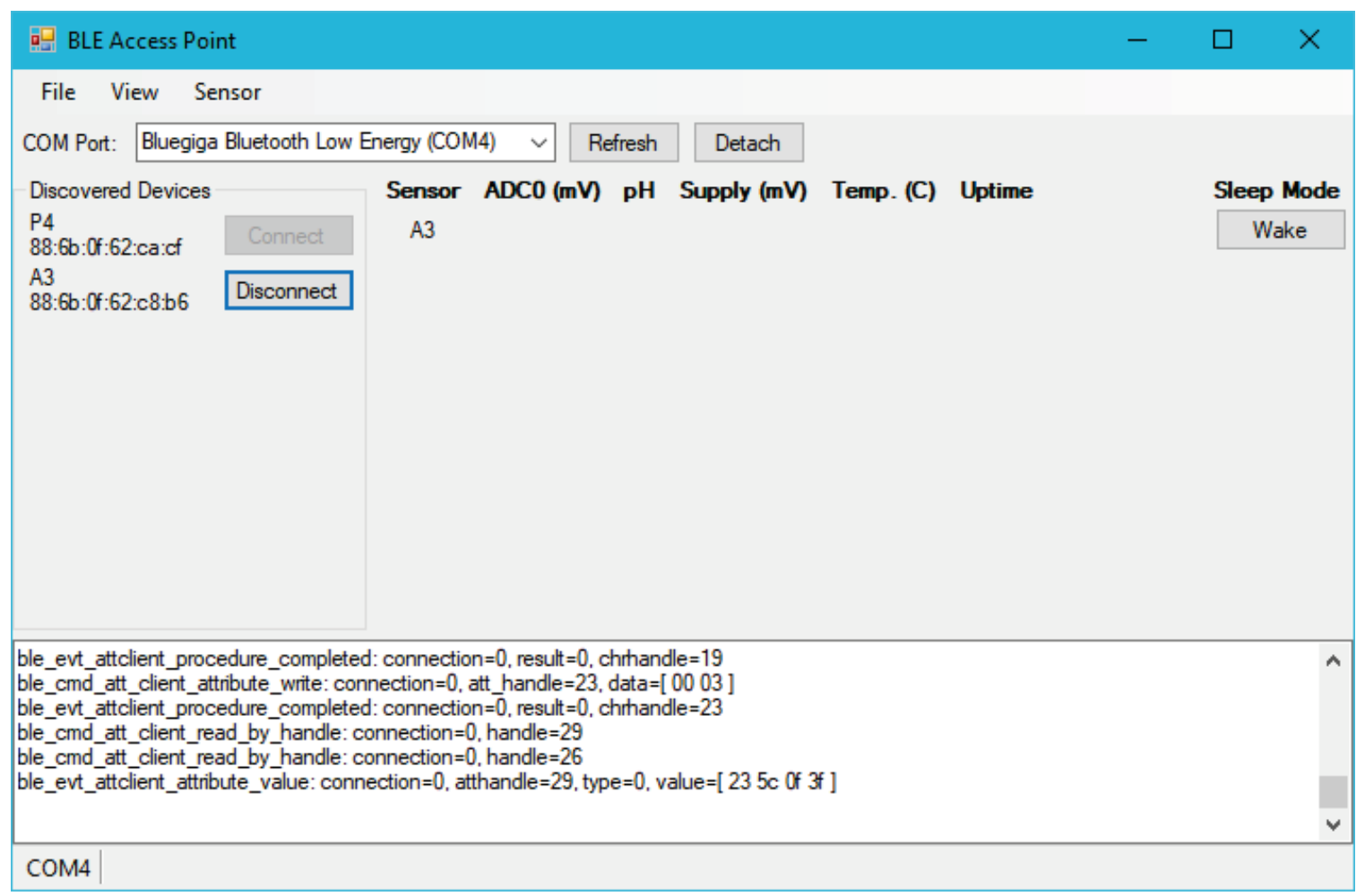

Figure 2.27. Device connected (sleep mode).

The Wake button is used to take the peripheral device out of sleep mode. When this is done, a CSV file is created containing the same header rows as those shown in the data table (except for Sleep Mode). As data is received from the peripheral device, it is written both to the CSV file and to the data table (shown in Figure 2.28). 


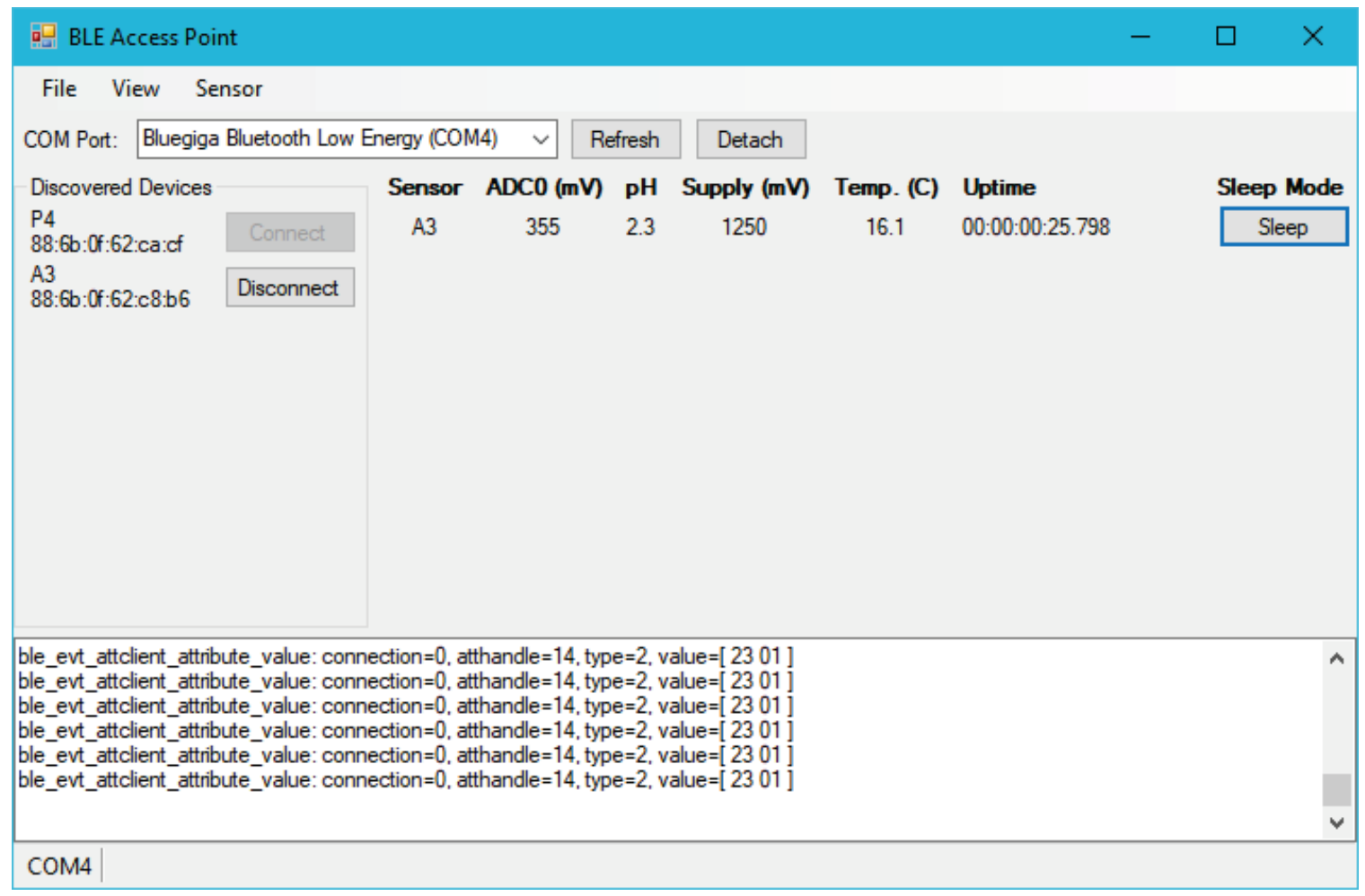

Figure 2.28. Device connected (wake mode).

An option to graph the most recent data is also available. Figure 2.29 shows the last ten seconds of data received by the sensor. This window of time may be adjusted to be longer if required by the application. 


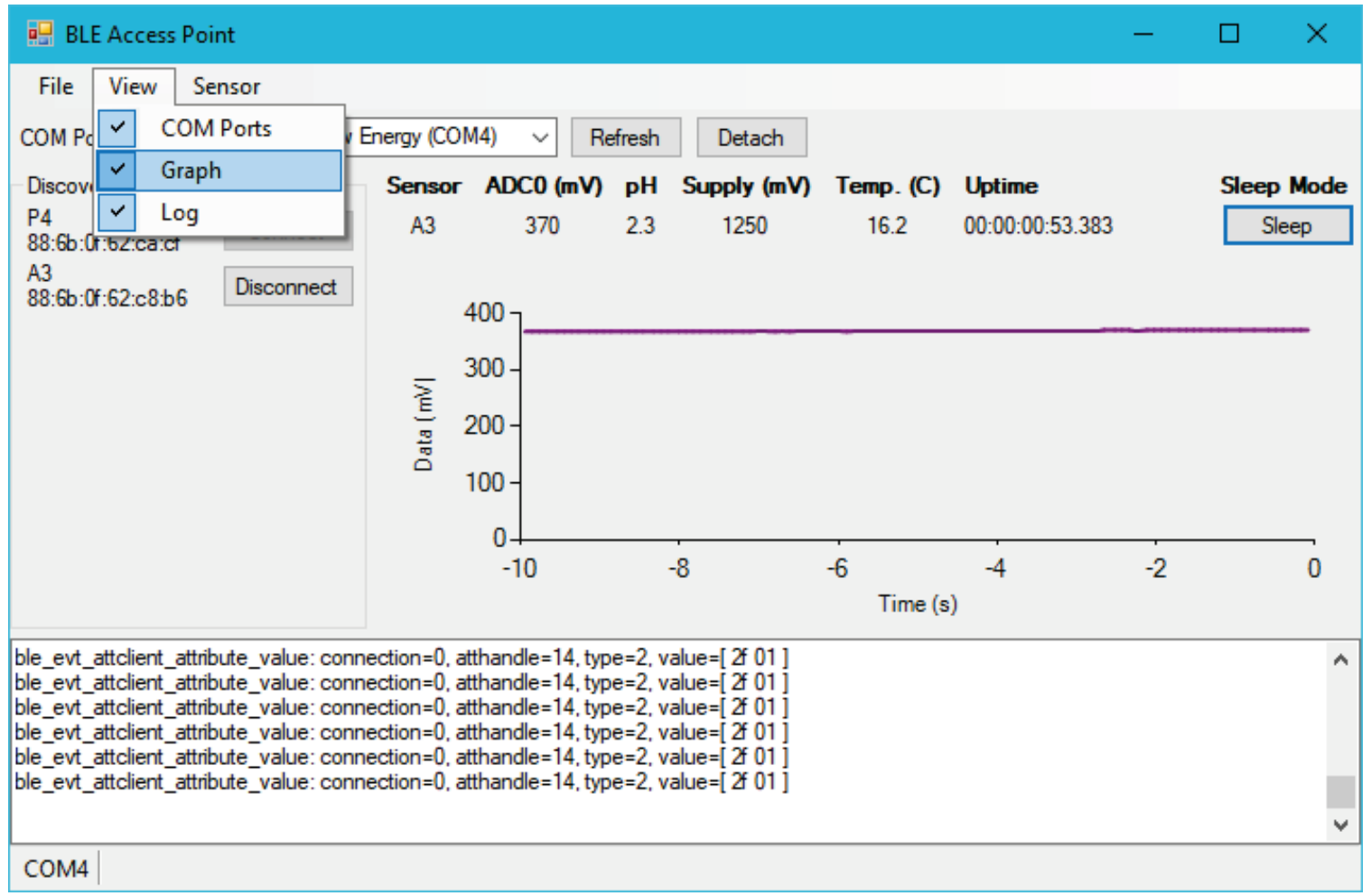

Figure 2.29. Device connected with graph enabled.

Varying offset values of the reference electrodes can also be calibrated using the software (Figure 2.30). To calibrate, the sensor may be placed in a solution with a known $\mathrm{pH}$, and that known $\mathrm{pH}$ and the voltage measured by the device will be used by the software to calculate the offset. This offset is then transmitted back to the peripheral device to be stored in flash memory. This offset is requested by the software during the connection process described above.

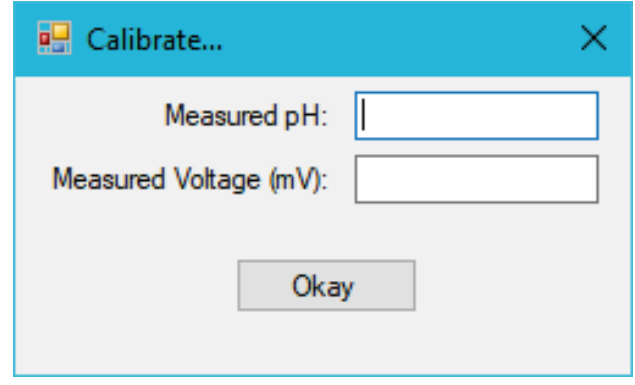

Figure 2.30. Calibration window.

Since the sensor platform is designed to be relatively inaccessible within the bioreactor, it is important to be able to recover from any errors that occur remotely. A magnetic switch is provided on the sensor platform itself to allow for remote resets, but another option is 
provided in the software. A Reset All function (Figure 2.31) performs a reset on the BLED112. When the central device is reset, it loses its connection to any devices connected with it, which causes the peripheral devices to reset themselves. A confirmation window is opened to prevent this from being performed accidentally by the user.

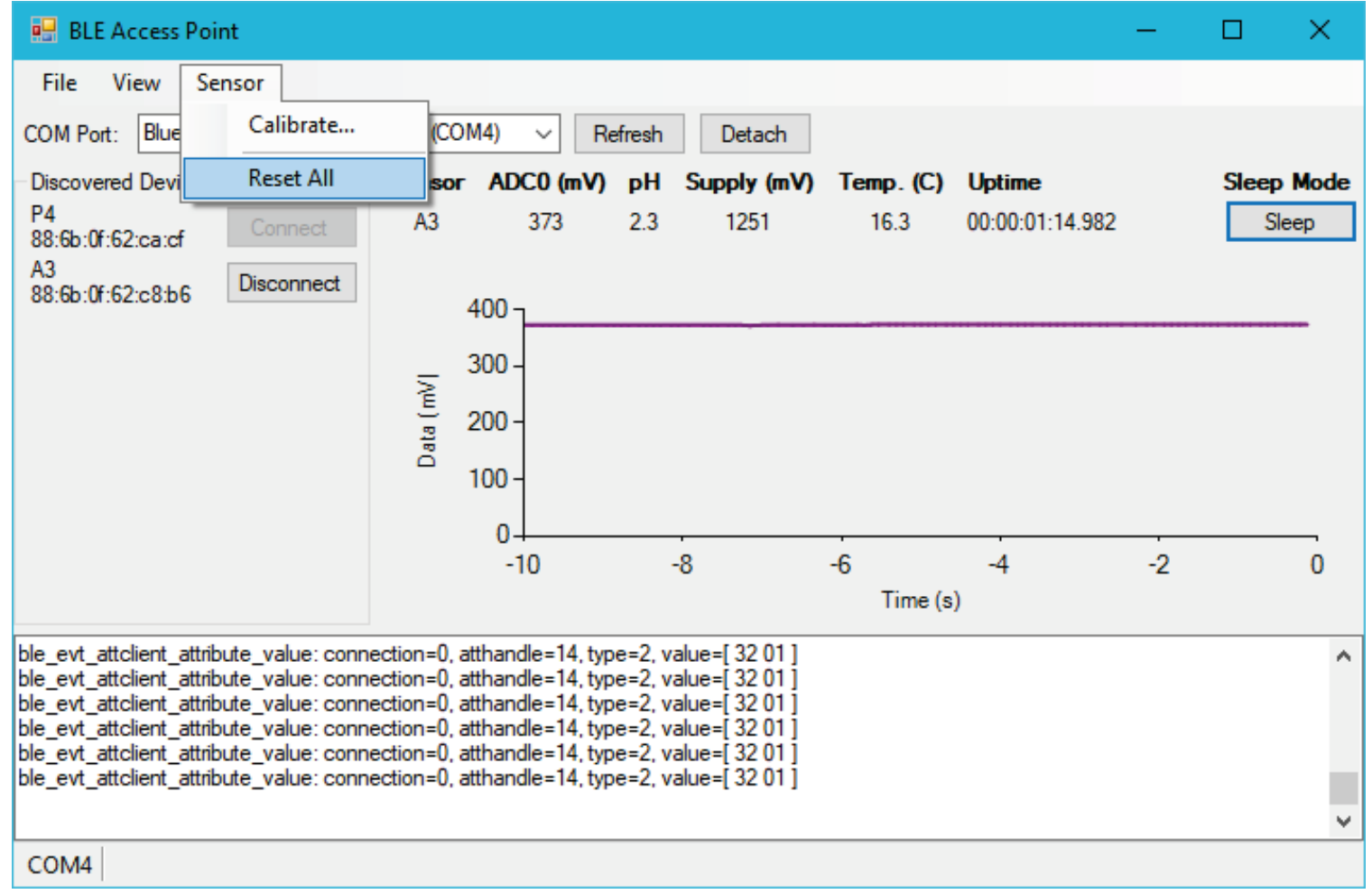

Figure 2.31. The Reset All function. 


\section{Validation Experiments}

\subsection{Methods}

Multiple benchtop experiments were performed to characterize the behavior of the $\mathrm{pH}$ sensors. While the most frequently used acidity range in mammalian cell cultures is between 7.0 to 8.0 [52], a wider range of buffer solutions were desired to demonstrate the

effectiveness of the sensor. To achieve this, a characterization test was performed to observe the behavior of the sensor from a $\mathrm{pH}$ of 4 to 10 . A benchtop response time test was also performed to characterize the delay that occurs as $\mathrm{pH}$ changes.

\subsection{1 pH Characterization}

Buffer solutions with a $\mathrm{pH}$ of $4,5,6,7,8,9$, and 10 were prepared using mixtures of $0.1 \mathrm{M}$ sodium hydroxide $(\mathrm{NaOH})$ and $0.1 \mathrm{M}$ monopotassium phosphate $\left(\mathrm{KH}_{2} \mathrm{PO}_{4}\right)$. In each trial, three different tantalum sensing electrodes were tested with a single silver-silver chloride reference electrode (Section 2.3.1) in each buffer solution. For example: reference electrode R1 and sensing electrode $\mathrm{S} 1$ were submerged in the $\mathrm{pH} 4$ solution. After the voltage was allowed to settle for two minutes, the voltage difference between the electrodes was recorded using a Keithley 2000 multimeter. Then, sensing electrode S1 was replaced in the solution by sensing electrode S2. Once this measurement was recorded, S2 was similarly replaced by sensing electrode S3. This was then repeated in the pH 5 buffer solution, and so on. A total of four reference electrodes were tested this way. Before each buffer solution was tested, its $\mathrm{pH}$ was measured using the BK Precision $760 \mathrm{pH}$ meter, and the buffer solution was adjusted when necessary. Both reference electrodes and sensing electrodes were rinsed in deionized water between immersions in different buffer solutions.

\subsubsection{Response Time Characterization}

A single fully assembled $\mathrm{pH}$ sensor with reference electrode and sensing electrode was assembled. This sensor was submerged in an acidic buffer solution with $\mathrm{pH} 4.49$ which was constantly being mixed using a magnetic stirrer. The BK Precision $760 \mathrm{pH}$ meter was also submerged at the same time so that both sensors worked simultaneously. The voltage 
measured by the $\mathrm{pH}$ sensor was recorded using a Keithley 2000 multimeter and logged to a computer. After allowing two minutes of settling time, $1 \mathrm{~mL}$ of $\mathrm{NaOH}$ was titrated into the solution. Once the measurements had settled, another $\mathrm{mL}$ of $\mathrm{NaOH}$ was added to the solution, and another, until four increases in acidity had been performed in the same manner.

\subsection{Results}

\subsection{1 pH Characterization}

The results of the $\mathrm{pH}$ characterization test are displayed in Figure 3.1, where the measured voltage is the voltage of the reference electrode minus the voltage of the sensing electrode. Each reference electrode (R1 to R4) are shown separately to demonstrate the differences between them. Different sensing electrodes (S1 to S3) are not displayed separately since there was no significant trend found between these electrodes. A linear model is also displayed for each reference electrode. 


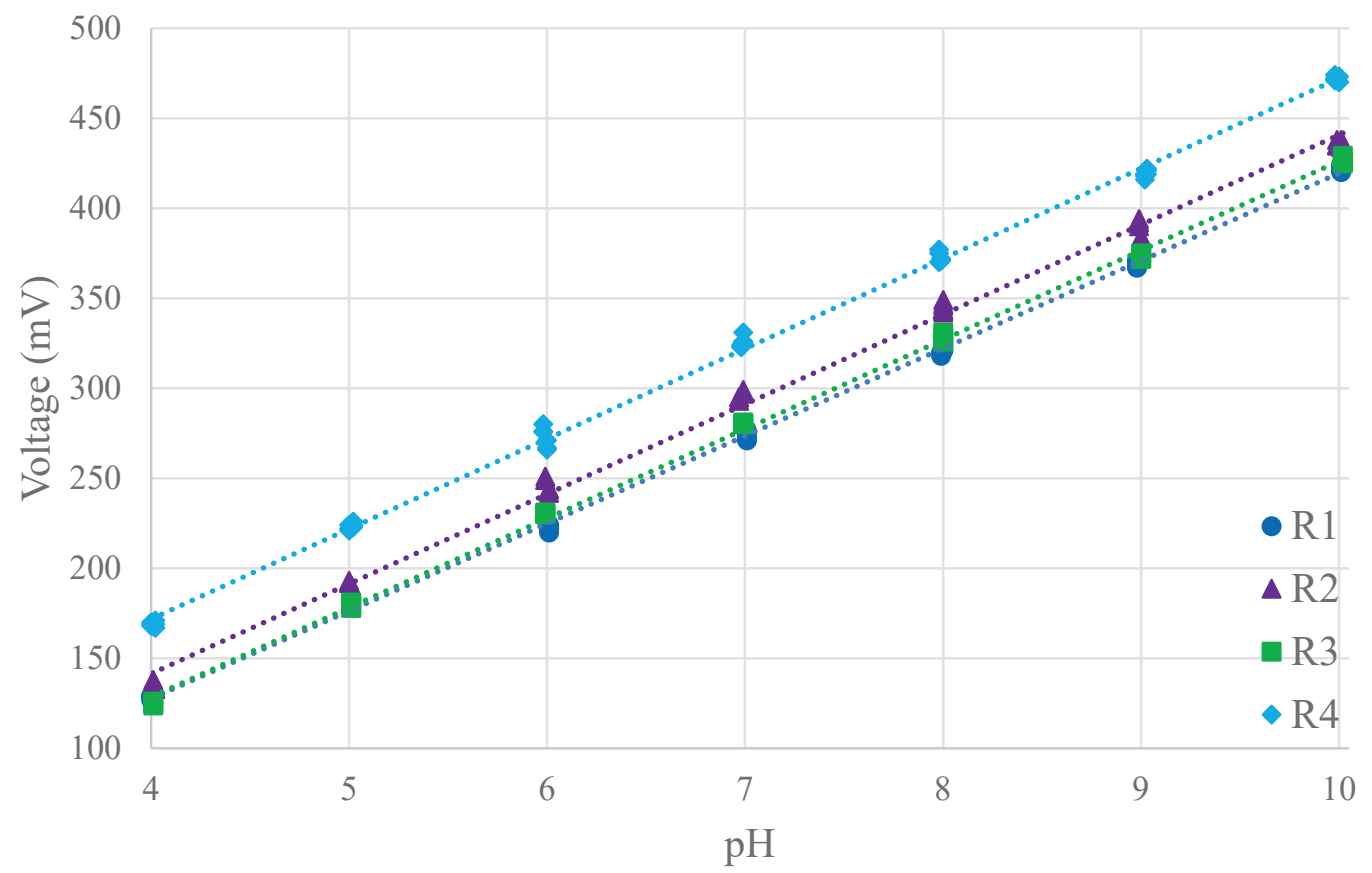

Figure 3.1. pH sensor characterization data.

The parameters found to describe the linear model are shown in Table 3.1A. The number of data points varies between trials $(\mathrm{R} 1: \mathrm{N}=36 ; \mathrm{R} 2: \mathrm{N}=42 ; \mathrm{R} 3: \mathrm{N}=21 ; \mathrm{R} 4: \mathrm{N}=$ 42). Table 3.1B shows the linear model derived from the data of the response time characterization test $(\mathrm{N}=5)$.

Table 3.1. Linear fit of sensor data for experiment 1 (A) and experiment 2 (B).

(A)

\begin{tabular}{|c|c|c|c|}
\hline & Slope $(\mathrm{mV} / \mathrm{pH})$ & Intercept (mV) & $\mathbf{R}^{2}$ \\
\hline R1 & 48.5981 & -66.6280 & 0.9990 \\
\hline R2 & 49.8619 & -57.8784 & 0.9969 \\
\hline R3 & 49.5917 & -69.6644 & 0.9991 \\
\hline \multirow[t]{2}{*}{ R4 } & 50.0406 & -28.2485 & 0.9985 \\
\hline & Slope $(\mathrm{mV} / \mathrm{pH})$ & Intercept (mV) & $\mathbf{R}^{2}$ \\
\hline R2-S4 & 48.6139 & -49.6502 & 0.9998 \\
\hline
\end{tabular}

\subsubsection{Response Time Characterization}

The results of the response time test are shown in Figure 3.2. The $\mathrm{pH}$ measurements shown correspond to the value recorded by the BK Precision $\mathrm{pH}$ meter when the reading had stabilized. Figure 3.3 shows each titration on a magnified scale to better show the 
response time. Based on this data, the $5 \%$ settling times $T_{s}$ for each titration sequentially are: $4.58 \mathrm{~s}, 5.29 \mathrm{~s}, 5.51 \mathrm{~s}$, and $6.14 \mathrm{~s}$.

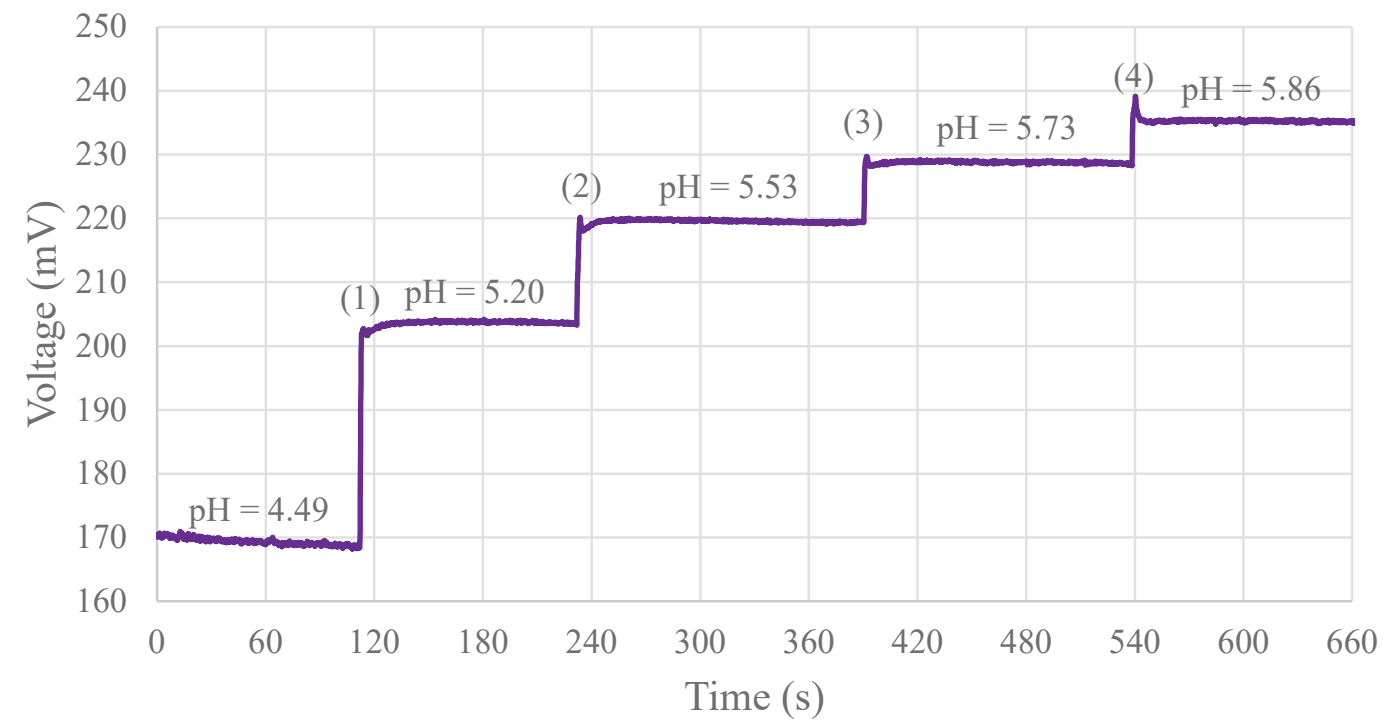

Figure 3.2. pH sensor response time data.

(1)

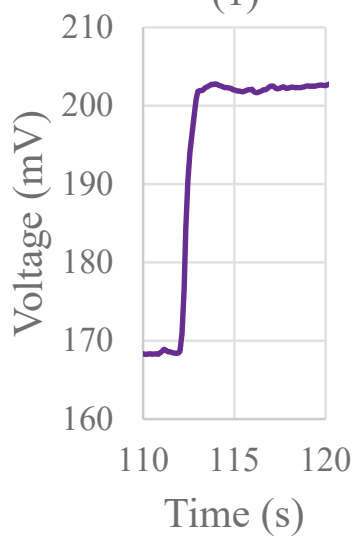

(2)

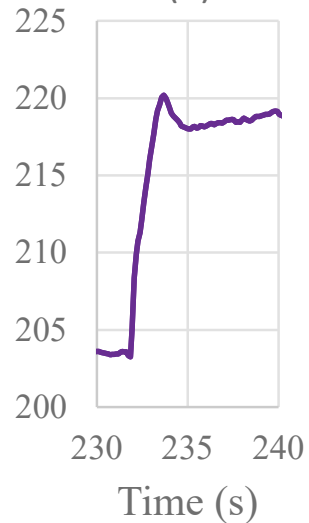

(3)

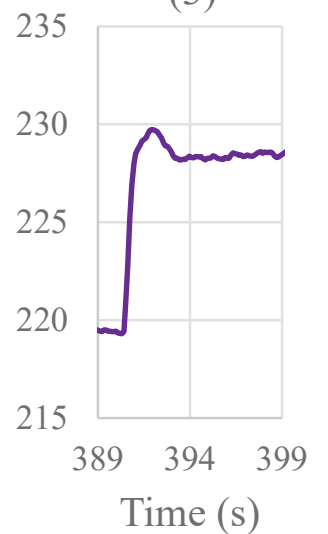

(4)

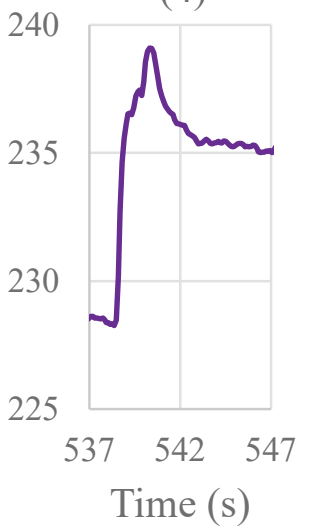

Figure 3.3. pH sensor response time data (titrations magnified).

\subsection{Discussion}

While the rate of change for the $\mathrm{pH}$ sensor remains very close (approximately 50 $\mathrm{mV} / \mathrm{pH}$ ) between different reference electrodes, the offset of each reference electrode can vary by over $40 \mathrm{mV}$. When the reference electrodes are compared with a commercially 
available silver-silver chloride reference electrode (Basi RE-6) they differ by about 20 to $60 \mathrm{mV}$. This range of offset values may be accounted for by providing a method for sensor calibration. In the case of this sensor platform, the slope of the $\mathrm{pH}$ response curve is a constant in the access point software, while the offset used in the calculation is stored in the flash memory of each sensor and may be provided to the access point.

The closeness of the data to the fitted model varies between trials. Trials with lower $\mathrm{N}$ show higher $\mathrm{R}^{2}$ values, where the data for the sensor response time test has the highest with $\mathrm{N}=5$. One source of measurement error may be the settling time. This settling time is relatively short when $\mathrm{pH}$ sensor was submerged in the solution during the $\mathrm{pH}$ change, but settling time was much slower when an electrode is first put into the liquid. In most cases, two minutes was sufficient for settling, but in other cases in the $\mathrm{pH}$ characterization test the measurement was still stabilizing when the measurement was recorded. This variation in settling time did not appear to correlate with different electrodes, and the cause of this variation is unknown.

The response time test shows that the electrodes have a 5\% settling time of approximately 5 seconds. This number increases as the change in $\mathrm{pH}$ decreases. For the smaller increases in $\mathrm{pH}$, the $\pm 5 \%$ window becomes wider, which may contribute to the slower settling time. Greater overshoot was also present in these cases, which may be due to differing diffusion behavior as the relative concentrations of $\mathrm{pH}$ became more similar. 


\section{Conclusion}

This thesis has presented a Bluetooth-based wireless sensor platform for in vivo applications. In particular, this technology has been presented for monitoring the acidity and temperature of a cell culture within a sealed bioreactor. The use of the Bluetooth LowEnergy standards provides bidirectional communication between the wireless sensor node and an access point so that the sensor node can send data to the access point and the access point can control and calibrate the sensor. Design of both the sensor node and access point have been discussed.

The next step of this project will be to implement the packaged sensor platform in a full submersion test inside a bioreactor. This test will provide information about the stability of the sensors, battery lifetime, and integrity of the sensor packaging, as well as further demonstrate the practicality of this sensor.

For bioreactor monitoring, the power from the selected internal battery may not be sufficient for continuous monitoring over the whole duration of cell manufacturing. The first modification will be to the wireless powering system. The commercially available wireless charging incorporated in this design works well for charging the sensor between tests without opening the packaging. However, the transmission range of WPC certified transmitters is limited to less than one centimeter. The use of resonant magnetic charging may help to give the additional range needed to transmit through the bioreactor wall.

Another change that may be made is to use a lower-power microcontroller. Two modifications are currently planned to address this in the future designs. More energyefficient alternatives to the BLE113 exist. For example, Silicon Labs also produces the BGM113 and BGM121/123, two Bluetooth-compatible microcontrollers that use the ARM M4 instead of the Intel 8051 and are advertised as consuming only half the current of the BLE113 during transmission [53-55]. The impact of this change will likely be small, since much of the power is used by the sensors. However, the BGM121 in particular may increase the transmission range of the sensor and allow for mesh networking.

Future work will also be needed to fully realize the greater scope of the project. The limited scope of this work includes monitoring of $\mathrm{pH}$ and temperature levels, but oxygen, glucose, and pressure are also important factors in the expansion process. As this project 
is continued, each of these sensing needs will be addressed. A reasonable next step may be the creation and miniaturization of a Clark electrode-style oxygen sensor to be incorporated into the sensor packaging.

Monitoring of conditions inside of a bioreactor is a powerful method of controlling cell growth, but they do not provide a measurement of cell potency. One method that has been used to provide an assay of cell potency is impedance spectroscopy [56, 57]. Multiple researchers have developed impedimetric sensors for use within the bioreactor environment which utilize impedance spectroscopy $[58,59]$. Impedimetric sensors of this nature may be included in future implementations of this sensor to provide valuable information about the potency of the cell culture. 


\section{Bibliography}

1. Sakthipriya, N., An effective method for crop monitoring using wireless sensor network. Middle-East Journal of Scientific Research, 2014. 20(9): p. 1127-1132.

2. Ruiz-Garcia, L., et al., A review of wireless sensor technologies and applications in agriculture and food industry: state of the art and current trends. sensors, 2009. 9(6): p. 4728-4750.

3. Mukhopadhyay, S.C., Wearable sensors for human activity monitoring: A review. IEEE sensors journal, 2015. 15(3): p. 1321-1330.

4. Knaian, A.N., A wireless sensor network for smart roadbeds and intelligent transportation systems. 2000, Massachusetts Institute of Technology.

5. Meesookho, C., S. Narayanan, and C. Raghavendra. Collaborative classification applications in sensor networks. in Sensor Array and Multichannel Signal Processing Workshop Proceedings, 2002. 2002. IEEE.

6. Lynch, J.P. and K.J. Loh, A summary review of wireless sensors and sensor networks for structural health monitoring. Shock and Vibration Digest, 2006. 38(2): p. 91-130.

7. Rashvand, H.F., et al., Wireless sensor systems for space and extreme environments: a review. IEEE Sensors Journal, 2014. 14(11): p. 3955-3970.

8. Wang, W.-Q. and D. Jiang, Integrated wireless sensor systems via near-space and satellite platforms: A review. IEEE Sensors Journal, 2014. 14(11): p. 3903-3914.

9. Karl, H. and A. Willig, Protocols and architectures for wireless sensor networks. 2007: John Wiley \& Sons.

10. Krishnamachari, B., Networking wireless sensors. 2005: Cambridge University Press.

11. Hill, J.L. and D.E. Culler, Mica: A wireless platform for deeply embedded networks. IEEE micro, 2002. 22(6): p. 12-24.

12. Polastre, J., R. Szewczyk, and D. Culler. Telos: enabling ultra-low power wireless research. in Proceedings of the 4th international symposium on Information processing in sensor networks. 2005. IEEE Press. 
13. Burns, A., et al., SHIMMER ${ }^{\mathrm{TM}} A$ wireless sensor platform for noninvasive biomedical research. IEEE Sensors Journal, 2010. 10(9): p. 1527-1534.

14. Gutierrez, J.A., et al., IEEE 802.15. 4: a developing standard for low-power lowcost wireless personal area networks. IEEE network, 2001. 15(5): p. 12-19.

15. Nachemson, A., Lumbar intradiscal pressure: experimental studies on postmortem material. Acta Orthopaedica Scandinavica, 1960. 31(sup43): p. 1-104.

16. Rohlmann, A., et al., Monitoring the load on a telemeterised vertebral body replacement for a period of up to 65 months. European Spine Journal, 2013. 22(11): p. 2575-2581.

17. Rohlmann, A., et al., Spinal loads during post-operative physiotherapeutic exercises. PLoS One, 2014. 9(7): p. e102005.

18. Bergmann, G., et al., In vivo glenohumeral contact force--Measurements in the first patient 7 months postoperatively. Journal of Biomechanics, 2007. 40: p. 21392149 .

19. Gustke, K.A., et al., A Targeted Approach to Ligament Balancing Using Kinetic Sensors. J Arthroplasty, 2017. 32(7): p. 2127-2132.

20. Asada, H.H., et al., Mobile monitoring with wearable photoplethysmographic biosensors. IEEE engineering in medicine and biology magazine, 2003. 22(3): p. $28-40$.

21. Huang, X., et al., Stretchable, wireless sensors and functional substrates for epidermal characterization of sweat. small, 2014. 10(15): p. 3083-3090.

22. Zhao, X., et al. Towards low-power wearable wireless sensors for molecular biomarker and physiological signal monitoring. in Circuits and Systems (ISCAS), 2017 IEEE International Symposium on. 2017. IEEE.

23. Yee, C., et al., Adoptive $T$ cell therapy using antigen-specific $C D 8+T$ cell clones for the treatment of patients with metastatic melanoma: in vivo persistence, migration, and antitumor effect of transferred T cells. Proceedings of the National Academy of Sciences, 2002.99(25): p. 16168-16173. 
24. Rosenberg, S.A., et al., Durable complete responses in heavily pretreated patients with metastatic melanoma using T-cell transfer immunotherapy. Clinical Cancer Research, 2011. 17(13): p. 4550-4557.

25. Maude, S.L., et al., Chimeric antigen receptor $T$ cells for sustained remissions in leukemia. New England Journal of Medicine, 2014. 371(16): p. 1507-1517.

26. Lee, D.W., et al., T cells expressing CD19 chimeric antigen receptors for acute lymphoblastic leukaemia in children and young adults: a phase 1 dose-escalation trial. The Lancet, 2015. 385(9967): p. 517-528.

27. Maus, M.V., et al., Adoptive immunotherapy for cancer or viruses. Annual review of immunology, 2014. 32: p. 189-225.

28. Kaiser, A., et al., Towards a commercial process for the manufacture of genetically modified T cells for therapy. Cancer gene therapy, 2015. 22(2): p. 72-78.

29. Levine, B.L., Performance-enhancing drugs: design and production of redirected chimeric antigen receptor (CAR) T cells. Cancer gene therapy, 2015. 22: p. 79-84.

30. Wang, X. and I. Rivière, Clinical manufacturing of CAR T cells: foundation of a promising therapy. Molecular Therapy-Oncolytics, 2016. 3: p. 16015.

31. Smith, J.W., Apheresis techniques and cellular immunomodulation. Therapeutic Apheresis and Dialysis, 1997. 1(3): p. 203-206.

32. Becker, T., et al., Future Aspects of Bioprocess Monitoring, in White Biotechnology, R. Ulber and D. Sell, Editors. 2007, Springer Berlin Heidelberg: Berlin, Heidelberg. p. 249-293.

33. Restifo, N.P., M.E. Dudley, and S.A. Rosenberg, Adoptive immunotherapy for cancer: harnessing the T cell response. Nature Reviews Immunology, 2012. 12(4): p. 269-281.

34. Hagen, T. Novartis Sets a Price of \$475,000 for CAR T-Cell Therapy. 2017.

35. Levine, B.L., et al., Global manufacturing of CAR $T$ cell therapy. Molecular Therapy-Methods \& Clinical Development, 2017. 4: p. 92-101.

36. Bravery, C.A., et al., Potency assay development for cellular therapy products: an ISCT review of the requirements and experiences in the industry. Cytotherapy, 2013. 15(1): p. 9-19. 
37. Li, F., et al. Cell culture processes for monoclonal antibody production. in MAbs. 2010. Taylor \& Francis.

38. Wayte, J., et al., pH: Effects on growth and productivity of cell lines producing monoclonal antibodies: Control in large-scale fermenters. Genetic Engineer and Biotechnologist, 1997. 17(2-3): p. 125-132.

39. Nordon, A., et al., In situ monitoring of the seed stage of a fermentation process using non-invasive NIR spectrometry. Analyst, 2008. 133(5): p. 660-666.

40. Acha, V., et al., ATR-FTIR sensor development for continuous on-line monitoring of chlorinated aliphatic hydrocarbons in a fixed-bed bioreactor. Biotechnology and bioengineering, 2000. 68(5): p. 473-487.

41. Lee, H.L., et al., In situ bioprocess monitoring of Escherichia coli bioreactions using Raman spectroscopy. Vibrational Spectroscopy, 2004. 35(1-2): p. 131-137.

42. Boehl, D., et al., Chemometric modelling with two-dimensional fluorescence data for Claviceps purpurea bioprocess characterization. Journal of biotechnology, 2003. 105(1-2): p. 179-188.

43. Lourenço, N., et al., Bioreactor monitoring with spectroscopy and chemometrics: a review. Analytical and bioanalytical chemistry, 2012. 404(4): p. 1211-1237.

44. Bhadra, S., et al. Wireless passive sensor for $\mathrm{pH}$ monitoring inside a small bioreactor. in Instrumentation and Measurement Technology Conference (I2MTC), 2013 IEEE International. 2013. IEEE.

45. Vasala, A., et al., A new wireless system for decentralised measurement of physiological parameters from shake flasks. Microbial cell factories, 2006. 5(1): p. 8.

46. Che, X., et al., Re-evaluation of RF electromagnetic communication in underwater sensor networks. IEEE Communications Magazine, 2010. 48(12): p. 143-151.

47. Lloret, J., et al., Underwater wireless sensor communications in the $2.4 \mathrm{GHz} I S M$ frequency band. Sensors, 2012. 12(4): p. 4237-4264.

48. Bluetooth SIG, Bluetooth Core Specifications Version 4.0. 2010.

49. Horton, B.E., et al., A varactor-based, inductively coupled wireless $p H$ sensor. IEEE Sensors Journal, 2011. 11(4): p. 1061-1066. 
50. Analog Devices, $40 \mu A$ Micropower Instrumentation Amplifier with Zero Crossover Distortion. 2009.

51. Texas Instruments, LMT89 2.4-V, 10- $\mathrm{AA}$, SC70 Temperature Sensor. 2015.

52. Eagle, H., Buffer combinations for mammalian cell culture. Science, 1971. 174(4008): p. 500-503.

53. Silicon Labs, BLE113 Bluetooth Smart Module. 2015.

54. Silicon Labs, BGM113 Blue Gecko Bluetooth Module Data Sheet. 2016.

55. Silicon Labs, BGM121/BGM123 Blue Gecko Bluetooth SiP Module Data Sheet. 2017.

56. Seidel, D., et al., Impedimetric real-time monitoring of neural pluripotent stem cell differentiation process on microelectrode arrays. Biosensors and Bioelectronics, 2016. 86: p. 277-286.

57. K'Owino, I.O. and O.A. Sadik, Impedance spectroscopy: a powerful tool for rapid biomolecular screening and cell culture monitoring. Electroanalysis, 2005. 17(23): p. 2101-2113.

58. Kim, Y.-H., J.-S. Park, and H.-I. Jung, An impedimetric biosensor for real-time monitoring of bacterial growth in a microbial fermentor. Sensors and Actuators B: Chemical, 2009. 138(1): p. 270-277.

59. Mishima, K., A. Mimura, and Y. Takahara, On-line monitoring of cell concentrations during yeast cultivation by dielectric measurements. Journal of fermentation and bioengineering, 1991. 72(4): p. 296-299. 